\title{
Radiation damage effects in Si materials and detectors and rad-hard Si detectors for SLHC
}

\section{Z. $\mathbf{L i}^{1}$}

Instrumentation Division, Brookhaven National Laboratory, 20 Technology St., Upton, NY 11973-5000, U.S.A.

E-mail: zhengl@bnl.gov

ABSTRACT: Silicon sensors, widely used in high energy and nuclear physics experiments, suffer severe radiation damage that leads to degradations in sensor performance. These degradations include significant increases in leakage current, bulk resistivity, space charge concentration, and free carrier trapping. For LHC applications, where the total fluence is in the order of $1 \times 10^{15} \mathrm{n}_{\mathrm{eq}} / \mathrm{cm}^{2}$ for 10 years, the increase in space charge concentration has been the main problem since it can significantly increase the sensor full depletion voltage, causing either breakdown if operated at high biases or charge collection loss if operated at lower biases than full depletion. For LHC Upgrade, or the SLHC, however, whit an increased total fluence up to $1 \times 10^{16} \mathrm{n}_{\mathrm{eq}} / \mathrm{cm}^{2}$, the main limiting factor for Si detector operation is the severe trapping of free carriers by radiation-induced defect levels. Several new approaches have been developed to make Si detector more radiation hard/tolerant to such ultra-high radiation, including 3D Si detectors, Current-Injected-Diodes (CID) detectors, and Elevated temperature annealing.

KEYWORDS: Hybrid detectors, Neutron detectors (cold, thermal, fast neutrons), dE/dx detectors, Pixelated detectors and associated VLSI electronics

\footnotetext{
${ }^{1}$ Corresponding author.
} 


\section{Contents}

1 Introduction 1

2 Radiation induced degradations in Si sensors 4

2.1 Displacement radiation damage in $\mathrm{Si} \quad 4$

2.2 Increase in sensor leakage current 5

2.3 Damage in sensor electrical neutral bulk 5

2.4 Space charge transformations and CCE loss $\quad 7$

$\begin{array}{lll}\text { 2.4.1 Space charge transformation in as-irradiated Si sensors } & 7\end{array}$

2.4.2 Space charge transformation with annealing time and temperature 8

2.4.3 Space charge transformation due to free carrier trapping (space charge manipulation) 10

$\begin{array}{ll}\text { 2.4.4 Double junction/double peak electric field distribution } & 12\end{array}$

3 Radiation hardness of Si sensors - Previous approaches for LHC 13

3.1 Material/Impurity/Defect Engineering (MIDE) 13

3.2 Device Structure Engineering (DSE) 15

$\begin{array}{lll}\text { 3.2.1 Multi-guard ring system } & 15\end{array}$

3.2.2 Semi-3D detectors 16

3.2.3 3D detectors 18

3.2.4 Thin detectors 18

4 Possible solutions for radiation tolerant Si detectors for SLHC 19

$\begin{array}{lll}4.1 & 3 \mathrm{D} \text { detectors in various configurations } & 19\end{array}$

4.2 Current-Injected-Diode detectors 22

4.3 Elevated temperature annealing 25

4.4 Summary 26

\section{Introduction}

For Si detector applications in current high energy physics experiments, such as those in LHC at CERN, the main effort has been directed so far towards the improvement (or reduction) of the detector full depletion voltage [1], which leads to the increase of detector sensitive volume and therefore the detector charge collection efficiency (CCE). This remains true if the level of radiation environment is in the order of $1 \times 10^{15} \mathrm{n}_{\mathrm{eq}} / \mathrm{cm}^{2}$, when the trapping of free carriers by radiation-induced defects are not significant $(<50 \%)$. However, for LHC upgrade, the SLHC, the expected radiation level will be 
10 times higher, up to $1 \times 10^{16} \mathrm{n}_{\mathrm{eq}} / \mathrm{cm}^{2}$. In this case, in addition to the detector full depletion problem, the trapping problem is now one of the main limiting factors to CCE.

The total charge collected by a detector, $Q$, can be considered to be a product of two terms [2]:

$$
Q=Q_{0} \cdot C C E_{G F} \cdot C C E_{t} \equiv Q_{0} \cdot \vartheta \cdot \eta
$$

The term $C C E_{G F}=\vartheta$ in eq. (1.1) is the geometrical factor, that is related to the detector depletion thickness $w$, the waiting function (detector segmentation scheme) $[3,4]$, and the detector configuration (e.g. planar detectors or $3 \mathrm{~d}$ detector) [5]. The term $C C E_{t} \equiv \eta$ is the trapping factor. For a simple planar pad detector with no segmentation, the weighting field $E_{w}$ is $1 / d$ [3], and the induced current by a moving charge $Q_{0}^{\prime}(t)$ is [6]:

$$
i(t)=Q_{0}^{\prime}(t) \cdot E_{w} \cdot V_{d r}(t)=Q_{0}^{\prime}(t) \cdot \frac{1}{d} \cdot v_{d r}(t)
$$

where $v_{d r}(t)$ is the carrier drift velocity that depends on the detector internal electric field, and $Q_{0}^{\prime}(t)$ is affected by trapping (for large detrapping constant) [6]:

$$
Q_{0}^{\prime}(t)=Q_{0} \cdot e^{-t / \tau_{t}}
$$

Where $\tau_{t}$ is the trapping time constant that is proportional inversely to the trap concentration and the total collected charge in eq. (1.1) can be rewritten as, using eq. (1.2) and (1.3):

$$
Q=\int_{0}^{t_{d r}} i(t) d t=\int_{0}^{t_{d r}} Q_{0} \cdot e^{-t / \tau_{t}} \cdot \frac{1}{d} \cdot v_{d r}(t) d t
$$

Where $t_{d r}$ is the carrier transient time or drift time. For simplicity, we assume a constant electric field and therefore constant carrier drift velocity $V_{d r}$, we have:

$$
Q=Q_{0} \cdot \frac{w}{d} \cdot \frac{\tau_{t}}{t_{d r}}\left(1-e^{-t_{d r} / \tau_{t}}\right), \quad \text { with } \quad \theta=\frac{w}{d} \quad \text { and } \quad \eta=\frac{\tau_{t}}{t_{d r}}\left(1-e^{-t_{d r} / \tau_{t}}\right)
$$

where $w$ is the detector full depletion depth, and the expression $w=V_{d r} \cdot t_{d r}$ is used for planar detectors.

If $Q_{0}$ is the total charge deposited by a MIP particle in a detector of the thickness of $d$, then it can be expressed in terms of the number of electrons as the following:

$$
Q_{0}=80 \cdot d \quad(\text { Electrons, here } d \text { is in } \mu \mathrm{m})
$$

and eq. (1.5) can be rewritten as:

$$
Q=80 w \cdot \frac{\tau_{t}}{t_{d r}}\left(1-e^{-t_{d r} / \tau_{t}}\right) \quad(\text { Electrons, herewis in } \mu \mathrm{m})
$$

For situation with large trapping, such as in the SLHC with up to $10^{16} \mathrm{n}_{\mathrm{eq}} / \mathrm{cm}^{2}$ radiation, $\frac{\tau_{t}}{t_{d r}} \ll 1$, we have:

$Q \cong 80 w \cdot \frac{\tau_{t}}{t_{d r}}=80 \cdot v_{d r} \cdot \tau_{t} \equiv 80 \cdot d_{C C E} \quad$ (Electrons, here $d_{C C E}$ is in $\left.\mu \mathrm{m}\right) \quad\left(\right.$ very high fluences, $\left.\frac{\tau_{t}}{t_{d r}} \ll 1\right)$ 
where $d_{C C E}=v_{d r} \cdot \tau_{t}$ is defined as the charge collection distance (or trapping distance) and it is in the order of $20 \mu \mathrm{m}$ for $10^{16} \mathrm{n}_{\mathrm{eq}} / \mathrm{cm}^{2}$ radiation if one uses the trapping time constant of about $0.2 \mathrm{~ns}$, and saturation drift velocity of $10^{7} \mathrm{~cm} / \mathrm{s}$ for $v_{d r}$ [7] . It is clear that at very high fluences, the collected charge depends only on the charge collection distance that is usually much less than the detector thickness. In this case, it does not matter if a detector with thickness of $d \geq 50 \mu \mathrm{m}$ is fully depleted or not, the collected charge is about the same if $w \geq d_{C C E}$, and it is in the order of 1600 e's for $10^{16} \mathrm{n}_{\mathrm{eq}} / \mathrm{cm}^{2}$ radiation, regardless of the detector thickness $\left(d \geq d_{C C E}\right)$.

For low or modest radiation fluences, where $\frac{\tau_{t}}{t_{d r}} \gg 1$, eq. (1.7) can be re-written as:

$$
Q=80 \mathrm{w} \cdot\left(1-\frac{1}{2} \cdot \frac{t_{d r}}{\tau_{t}}\right) \quad \text { (Electrons) } \quad\left(\text { low or modest fluences, } \frac{\tau_{t}}{t_{d r}} \gg 1\right)
$$

In this case, the detector depletion depth $w$ is still an important factor in the collected charge, which depends on the bias voltage:

$$
w=\sqrt{\frac{2 \varepsilon \varepsilon_{0} V}{e N_{\mathrm{eff}}}}
$$

and relates to drift time via $w=v_{d r} \cdot t_{d r}$, where $e$ is the electronic charge, $\varepsilon$ the permittivity of $\mathrm{Si}, \varepsilon_{0}$ the permittivity of vacuum, $V$ the bias voltage, and $N_{\text {eff }}$ the effective space charge density.

The trapping $\left(\tau_{t}\right)$ and detrapping $\left(\tau_{d}\right)$ time constants for a trap level can be defined by:

$$
\left\{\begin{array}{l}
\tau_{t}^{=} \frac{1}{\sigma v_{\mathrm{th}} N_{t}} 1 \\
\tau_{d}^{=} \frac{1}{\sigma v_{\mathrm{th}} N_{C} e^{-E_{t} / k T}}
\end{array}\right.
$$

where $\sigma$ is the capture cross section of the trap, $v_{\text {th }}$ is the thermal velocity of charge carriers, $N_{t}$ is the concentration of traps, $N_{C}$ the electric state density in the conduction band, and $E_{t}$ the trap energy level in the band gap.

The trapping time constant is nearly independent on temperature (or weak dependence on $T$ ). However, it depends strongly on the radiation fluence $\Phi_{n}$ through $N_{t}$. At a fluence of $10^{16} \mathrm{n}_{\mathrm{eq}} / \mathrm{cm}^{2}$, the trapping time can be as short as $0.2 \mathrm{~ns}$ [7]. It is obvious that to improve detector charge collection in the SLHC environment, in addition to improving the detector depletion depth $w$, one needs to reduce the effect of trapping. There are a number of ways to do that:

1. Reducing the free carrier drift time $t_{d r}$ by making the detector thinner so that $\frac{\tau_{t}}{t_{d r}} \ll 1$ would not be the condition. In fact, for $\frac{\tau_{t}}{t_{d r}}=1$, the detector thickness would be about $20 \mu \mathrm{m}$ at a fluence of $10^{16} \mathrm{n}_{\mathrm{eq}} / \mathrm{cm}^{2}$. However, the total charger deposited by MIP in this thin detector would only be 1600 e's even before radiation. Therefore, the approach of thin detectors may improve the relative $\operatorname{CCE}\left(Q / Q_{0}\right)$, the absolute collected charge will not be increased;

2. Reducing the free carrier drift time $t_{d r}$ without reducing the detector thickness by decoupling the drift length and the detector thickness. This can be done by the 3D Si detectors in which columns of electrodes are etched into the Si wafer thickness, and free carriers are drifting between the electrodes columns laterally. Since the column spacing $\lambda_{C}$ can be made small, in the 
order of 40-100 $\mu \mathrm{m}, \frac{\tau_{t}}{t_{d r}} \cong 1$ can be satisfied for reduced trapping, and total induced charge by MIP is still $80 d$, which is 24,000 e's for a $300 \mu \mathrm{m}$ thick wafer.

In fact, we can estimate the collected charge in a 3D detector. The weighting field in a 3D detector is roughly $1 / \lambda_{C}$, eq. (1.7) can be re-written (using eq. (1.5) and (1.6)) as the following:

$$
Q_{3 D}=80 \frac{d}{\lambda_{C}} w \cdot \frac{\tau_{t}}{t_{d r}}\left(1-e^{-t_{d r} / \tau_{t}}\right)
$$

and eq. (1.8) can be re-written as:

$$
Q_{3 D} \cong 80 \frac{d}{\lambda_{C}} w \cdot \frac{\tau_{t}}{t_{d r}}=80 \frac{d}{\lambda_{C}} \cdot d_{C C E} \quad \text { (Electrons) (veryhighfluences) }
$$

It is clear that the collected charge in a 3D detector is amplified by a factor of $d / \lambda_{C}$, as compared to standard planar detectors. For a $300 \mu \mathrm{m}$ thick 3D detector with $50 \mu \mathrm{m}$ column spacing, this amplification is 6 !

1. Reducing the trapping itself by making the trapping centers neutral. This can be done by filling the traps with injected current and/or charges so that they are no longer active in trapping MIP induced free carriers. This is the concept of the Current Injected Diode (CID) detectors. We will discuss more of CID in detail later in the paper.

2. Reducing the trapping centers by removing them via elevated temperature annealing. This approach is called Detector Recovery and Improvement Via Elevated-temperature-annealing (DRIVE). Details will be given later in the paper as well.

\section{Radiation induced degradations in Si sensors}

\subsection{Displacement radiation damage in $\mathrm{Si}$}

It is well known that neutrons, protons, electrons, and even gamma radiation can lead to displacement damage in Si. The displacement damage is caused by displacement of a Si atom from its substitution site to an interstitial site to form a Frenkel pair, as shown in figure 1 for neutron situation. In the case of neutron radiation, due to the high Si recoil energy $(133 \mathrm{keV})$, the displacement damage is a cascade with many interactions, resulting in an extended damage region, or defect clusters [8]. In the case of gamma radiation, however, the displacement damage is caused by the Compton electrons (about $1 \mathrm{MeV}$ in energy) that only produce isolated single defects when vacancies/interstitials react to each other or to impurities in the $\mathrm{Si}$, as shown in figure 1. In the case of charged particles, protons, pions, etc., due to Coulomb interaction, the displacement damages in Si are partially defect clusters and partially isolated single defects. Therefore, even for the same amount of non-ionizing energy loss (NEIL) in Si, defect structures caused by different radiation particles can be very different. Table I summarizes qualitatively the defect structures caused by different types of radiation particles. 


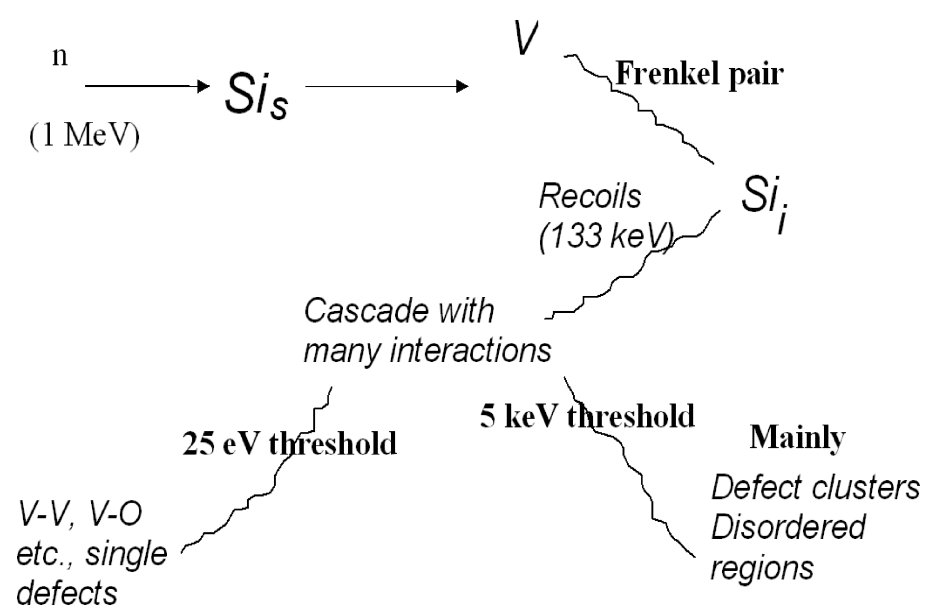

Figure 1. Primary and secondary defects in Si caused by fast neutrons.

\subsection{Increase in sensor leakage current}

Increase of sensor leakage current under radiation is caused by the radiation induced deep level defects that act as generation and recombination centers. These deep levels include V-V (double vacancy center) and V-V related complexes (clusters), P-V or E-center (phosphorus vacancy center P-V), O$\mathrm{V}$ or A-center (oxygen vacancy center) and levels in the defect clusters. It has been found that the leakage current density, current per unit volume, increases linearly with fluence. For $1 \mathrm{MeV}$ equivalent neutron fluence, $\Phi_{n, \text { equ }}$. The increase in volume current, $\Delta \mathrm{J}$, can be written as [9]:

$$
\Delta J=\alpha \cdot \Phi_{n, e q u}
$$

Where $\alpha$ is called the damage constant. The range of $\alpha$, for high resistivity $\operatorname{Si}(2-8 \mathrm{k} \Omega$-cm), is within $4-6 \times 10^{-17} \mathrm{~A} / \mathrm{cm}$ at $20{ }^{\circ} \mathrm{C}[10]$. Since the process of carrier generation via the deep levels is an activated process, the resulting leakage current is extremely sensitive to temperature: it decreases exponentially with decreasing temperature. This strong temperature dependence gives rise to the low temperature operation of Si sensors at greatly reduced leakage current. Figure 3 shows the leakage current dependence on temperature of a neutron irradiated Si sensor and simulated current using two deep levels [11]. It is clear that, a modest cooling from $300 \mathrm{~K}$ to $263 \mathrm{~K}\left(-10{ }^{\circ} \mathrm{C}\right)$ reduces the sensor leakage current by nearly 2 orders of magnitude. The fit to the total current using two deep levels agrees quite well to the experimental data [11]. The leakage current can be annealed at RT (room temperature) or at elevated temperatures. Figure 4 shows the annealing stages at RT of a neutron irradiated Si detector [12].

\subsection{Damage in sensor electrical neutral bulk}

When the sensor is not fully depleted, the un-depleted portion is at zero electric field, or neutral condition called electrical neutral bulk (ENB). Radiation induced changes in ENB are mainly in the carrier concentration, bulk resistivity, and carrier low field mobility. Radiation induced deep levels can cause carrier removal and compensation, leading to the dramatic increase in bulk resistivity. Figure 5 


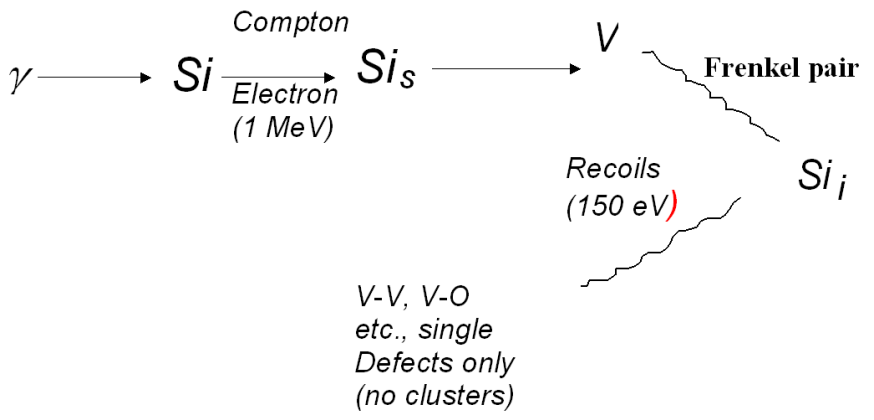

Figure 2. Primary and secondary defects in Si caused by gamma radiation.

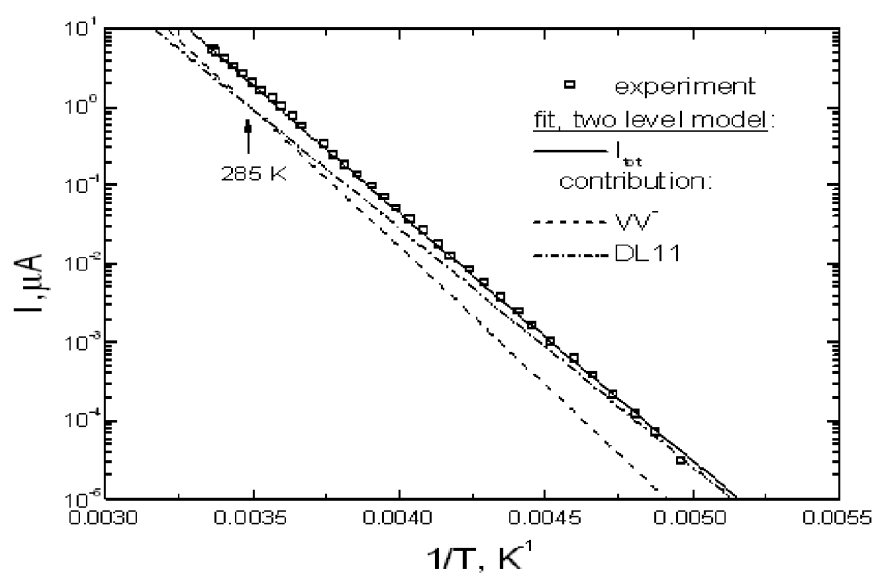

Figure 3. Leakage current as a function of temperature for n-irradiated Si sensors.

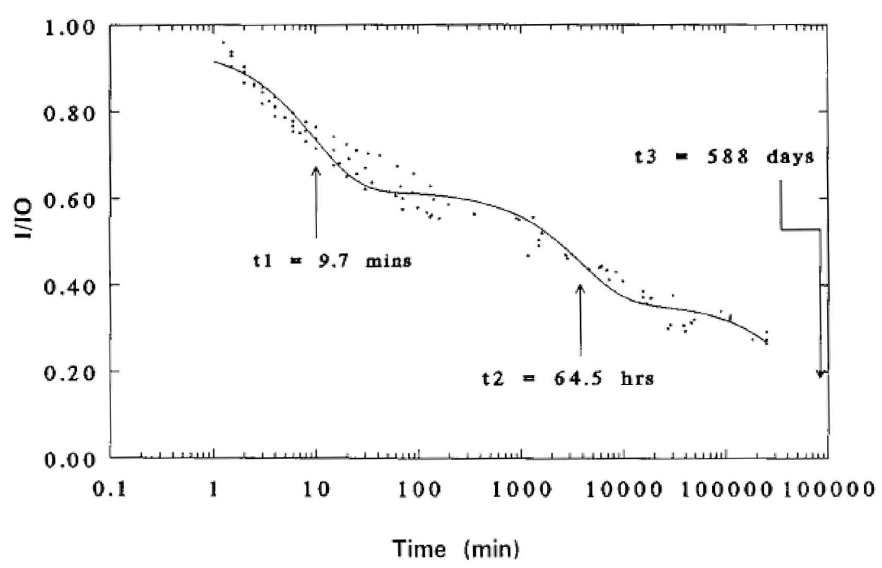

Figure 4. RT annealing of leakage current in n-irradiated Si sensors. 
Table 1. Different types of defect structures created by different radiation particles. Number of X's is a qualitative indication of the amount.

\begin{tabular}{|l|l|l|}
\hline Particle type & Single defects & Defect clusters \\
\hline $\mathrm{n}$ & $\mathrm{x}$ & $\mathrm{xxxxx}$ \\
\hline $\begin{array}{l}\text { Charged particles } \\
(\mathrm{p}, \pi, \text { etc. })\end{array}$ & $\mathrm{xxxx}$ & $\mathrm{xx}$ \\
\hline$\gamma, e$ & $\mathrm{xxxxxx}$ & \\
\hline
\end{tabular}

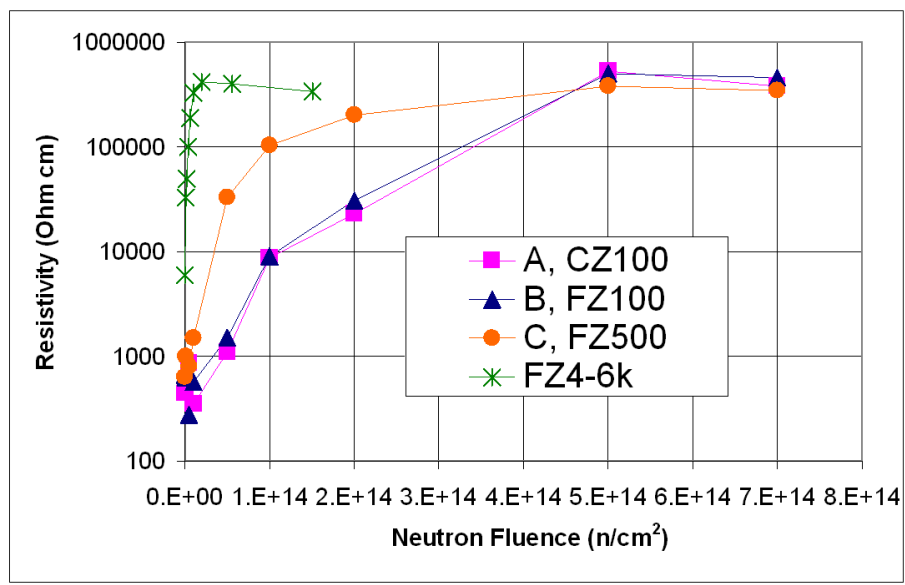

Figure 5. Changes in Si bulk resistivity with neutron fluence for various starting resistivity.

shows the ENB resistivity, obtained from a direct resistor measurement [13], as a function of neutron fluence for n-type Si materials with different resistivity. It is clear that ENB resistivity increases with neutron fluence regardless of Si initial resistivity and type ( $\mathrm{CZ}$ or FZ). The resistivity will eventually saturate at a value close the intrinsic resistivity, between $200-300 \mathrm{k} \Omega-\mathrm{cm}$. This effect is also confirmed by measurement using traditional Hall method [14]. Results in ref [14] also show that p-type Si material behaves similarly with n-type $\mathrm{Si}$, and the carrier Hall mobility degrades with radiation as well. The resistivity saturation with radiation was verified by the measurement of Fermi level position with radiation fluence [15], where Fermi level stabilization near the mid-band gap had been observed at high fluences.

\subsection{Space charge transformations and CCE loss}

\subsubsection{Space charge transformation in as-irradiated Si sensors}

For a fully depleted n-type Si sensor under radiation, the space charge sign undergoes a transformation from initially positive, to negative, termed as the space charge sign inversion (SCSI). This SCSI is caused by two processes: the donor removal (DR) and acceptor creation (AC). Figure 6 shows the data of the absolute value of the space charge concentration $\left(? \mathrm{~N}_{\mathrm{eff}}\right.$ ?) as a function of neutron fluence [16]. 


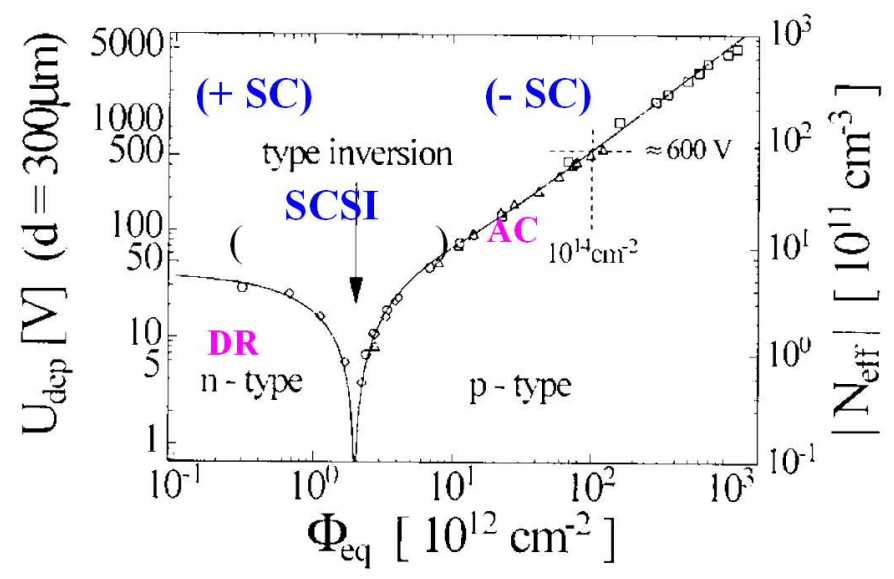

Figure 6. Changes in Si sensor full depletion voltage and S pace charge concentration with neutron fluences.

Figure 7. Cancellami

This space charge transformation in as radiated Si sensors can be described as the following:

$$
N_{\mathrm{eff}}=N_{d 0} \cdot e^{-\gamma \Phi_{n}}-\beta \Phi_{n} \cong-\beta \Phi_{n} \quad\left(\text { for } \Phi_{n} \geq 10^{14} \mathrm{n} / \mathrm{cm}^{2}\right)
$$

where $N_{d 0}$ is the initial shallow doping concentration, $\gamma$ the donor removal rate, and $\beta$ the introduction rate for deep acceptors. The first term in eq. (2.2) is DR, the second term is AC. Note that the parameter $N_{\text {eff }}$ has been given a sign freedom: at zero or low fluences, it is positive, and at high fluences, it is negative and dominated by the AC term. The specific fluence at which $N_{\text {eff }}$ becomes zero is called the SCSI fluence, or $\Phi_{i}$. The SCSI fluence has been found proportional to the initial shallow doping concentration $\left(N_{d 0}\right)$ of n-type $\mathrm{Si}$ sensor, and this leads an $N_{d 0}$ dependent donor removal rate, i.e.:

$$
\gamma=a / N_{d 0}
$$

as described in ref. [13] in a modified model. The typical value for $a$ is about 0.1 .

Since sensor full depletion voltage $\left(V_{\mathrm{fd}}\right)$ depends linearly on the absolute value of the space charge concentration:

$$
V_{\mathrm{fd}}=\frac{e d^{2}\left|N_{\mathrm{eff}}\right|}{2 \varepsilon \varepsilon_{0}} \cong \frac{e d^{2} \beta}{2 \varepsilon \varepsilon_{0}} \cdot \Phi_{n} \quad\left(\text { for } \Phi_{n} \geq 10^{14} \mathrm{n} / \mathrm{cm}^{2}\right)
$$

and it increases nearly linearly with fluence at high fluences, at a given operational bias, the detector may become partially depleted at high fluences. Since the typical value for $\beta$ is about $0.024 \mathrm{~cm}^{-1}$ [17], for a $300 \mu \mathrm{m}$ thick Si sensor, $V_{\mathrm{fd}}$ will increase to 173 volts after being irradiated to $1 \times 10^{14} \mathrm{n} / \mathrm{cm}^{2}$, much larger than the typical initial operation voltage of about 100 volts.

\subsubsection{Space charge transformation with annealing time and temperature}

It has been found that, the Si sensor space charge undergoes profound transformation at RT and elevated temperatures even after radiation being stopped. Figure 7 shows the sensor annealing behavior 
RT ANNEAL

$\mathrm{n}(111)$, 4-6 kohm-cm, Various oxides

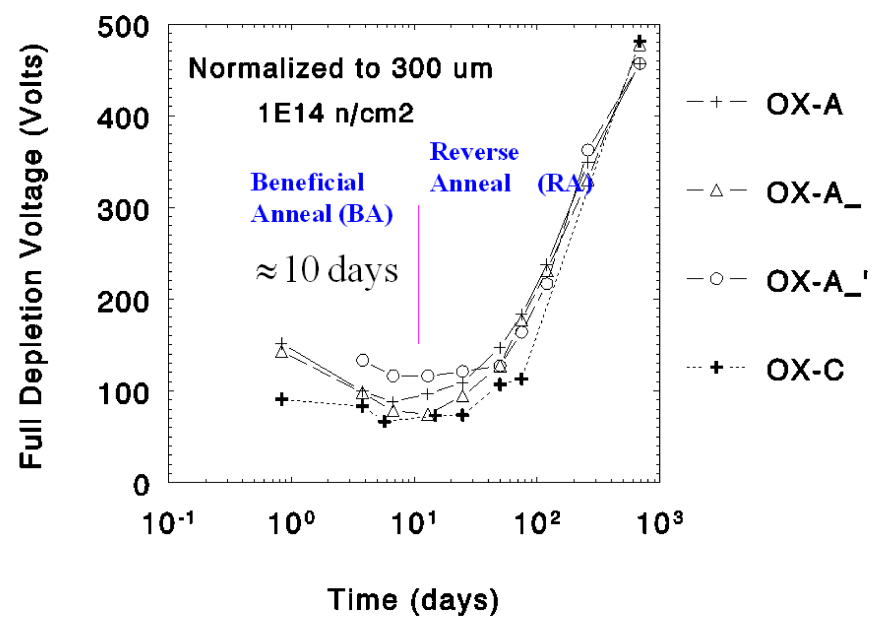

Figure 8. Beneficial and reverse annealing at RT in irradiated Si sensors fabricated from various thermal processes.

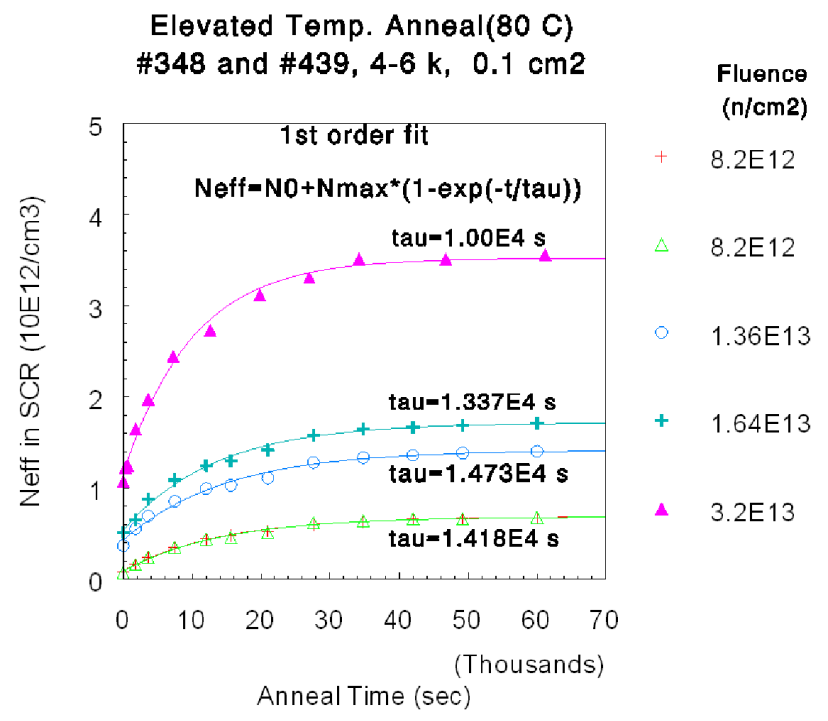

Figure 9. Reverse annealing at elevated temperatures for Si sensors irradiated to various fluences.

at RT after neutron radiation to a fluence of $1 \times 10^{14} \mathrm{n} / \mathrm{cm}^{2}$ for Si sensors processed at different conditions [18]. The sensors had all gone through the SCSI, and the normal annealing, termed as beneficial annealing, did occur within the first 10 days after radiation. During this period, the detector full depletion voltage decreases with annealing time, indicating a decrease in the absolute value of the space charge concentration. However, the sensor full depletion voltage starts to increase again after about 10 days annealing at RT. For those sensors shown in figure 7, the space charge was negative and became 
Table 2. Reverse annealing time constant at various temperatures.

\begin{tabular}{|ccccc|}
\hline $\mathrm{T}\left({ }^{\circ} \mathrm{C}\right)$ & -10 & 0 & 20 & 80 \\
\hline$\tau$ & $96.8 \mathrm{yr}$ & $14.6 \mathrm{yr}$ & $179 \mathrm{~d}$ & $3.7 \mathrm{hr}$ \\
\hline
\end{tabular}

less negative during the beneficial annealing period. After 10 days annealing at RT, the space charge became more negative. This increase of negative space charge was confirmed by the transient current technique for neutron irradiated Si sensors annealed at elevated temperature [19], and was termed as the "reverse annealing". Figure 8 shows the elevated temperature annealing of Si sensors irradiated by $1 \mathrm{MeV}$ neutrons to various fluences [20]. The reverse annealing process can be fitted to a first order process with multiple stages [20]. The one-stage first order fit is an average approach that gives practical parameters. Fittings to data in ref. $[18,20]$ give the following:

$$
N_{e f f}\left(t, \Phi_{n}\right)=N_{0}\left(\Phi_{n}\right)+N_{\mathrm{eff}}^{r, \max }\left(\Phi_{n}\right) \cdot\left(1-e^{-t / \tau(T)}\right)
$$

where $N_{0}\left(\Phi_{n}\right)$ is the initial space charge concentration before reverse annealing, $N_{\text {eff }}^{r, \max }\left(\Phi_{n}\right)$ the amplitude of reverse annealing ( $2^{\text {nd }}$ term in Equ. (2.5)), and $\tau(T)$ the reverse annealing time constant at a given temperature $(T)$. The values of $N_{0}\left(\Phi_{n}\right)$ and $N_{\mathrm{eff}}^{r, \max }\left(\Phi_{n}\right)$ are proportional to the fluence $(1 \mathrm{MeV}$ neutron equivalent) [20]:

$$
N_{0}\left(\Phi_{n}\right)=-0.035 \cdot \Phi_{n} ; N_{\mathrm{eff}}^{r, \max }\left(\Phi_{n}\right)=-0.073 \cdot \Phi_{n}
$$

and the reverse annealing process is an activated process [23]:

$$
\tau_{1 / 2}(T)=\tau(T) \cdot \ln 2=\frac{1}{v} \cdot e^{\frac{E_{a}}{k T}}
$$

where $\tau_{1 / 2}(T)$ is the time constant at which the reverse annealing reaches half of its amplitude, $v=$ $1 \times 10^{13} / \mathrm{s}$ is the jumping frequency, and $E_{a}=1.18 \mathrm{eV}$ is the activation energy.

It is clear that reverse annealing contributes significantly to the total space charge concentration. However, since it is an activated process, it is beneficial to operate the Si sensor at low temperatures to freeze out the reverse annealing. Table II lists the reverse annealing time constant at various temperatures. While it takes less than one year for reverse annealing to take place at RT, it can be delayed to about 15 years at modest cooling to $0{ }^{\circ} \mathrm{C}$. Si sensors to be used in LHC experiments are all planned to be operated at $-10{ }^{\circ} \mathrm{C}$ to $-20{ }^{\circ} \mathrm{C}$.

The mechanism of reverse annealing is believed to be related to the transformation of defect clusters during annealing [21]. During the reverse annealing period, defect clusters begin to break up, releasing single acceptor-like defects to the space charge region, which causes further increase in negative space charges. This model is consistent with the observation that, for gamma irradiated $\mathrm{Si}$ sensors where there is no defect clusters, there is no reverse annealing effect [22].

\subsubsection{Space charge transformation due to free carrier trapping (space charge manipulation)}

It has been observed that, for irradiated Si sensors, the space charge can be affected by the trapping status of free carriers injected into the sensor. In 1995, Eremin et al. first reported the space charge 


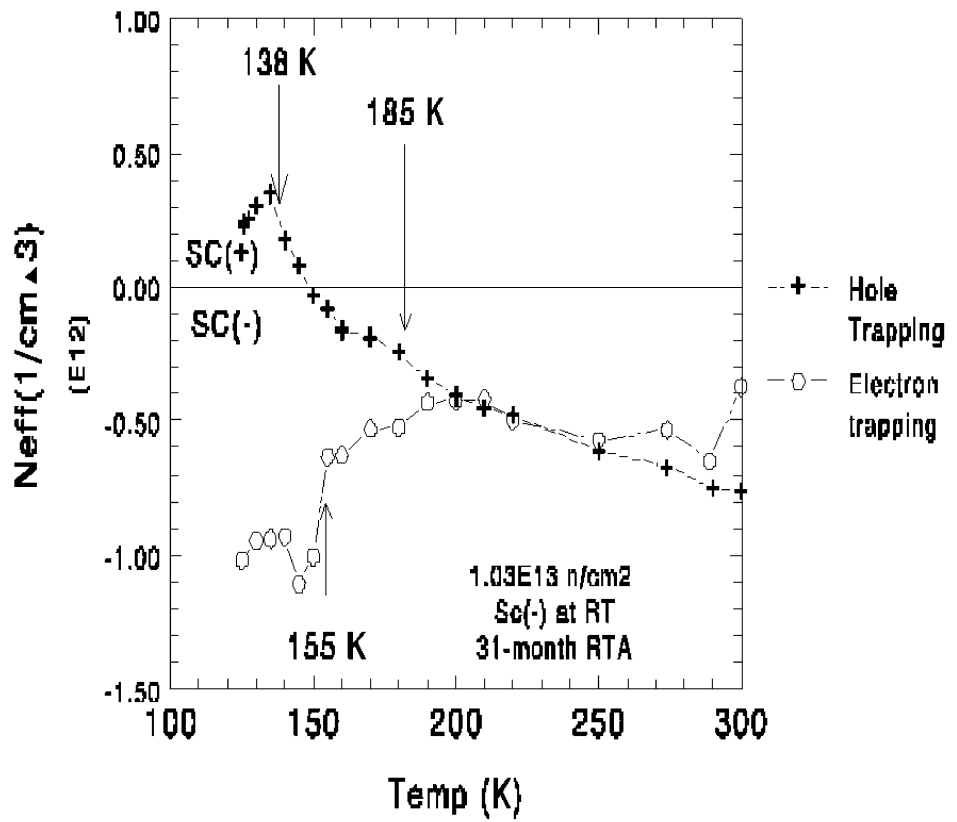

Figure 10. Free carrier trapping induced space charge changes in irradiated Si sensor at cryogenic temperatures.

transformation by laser induced free electrons and holes [23]. In this paper, it has been observed that hole trapping by radiation induced deep levels can cause the space charge sign "re-inversion": from its already inverted state of negative sign to positive sign after trapping of free holes introduced by laser. The space charge, both sign and concentration, can be therefore manipulated by injected free carriers. Data shown in Fig 10 come from direct measurements of space charge with temperature for both hole trapping and electron trapping [24]. The space charge was initially negative at RT $(300 \mathrm{~K})$, which was resulted from the radiation induced SCSI. With hole trapping, the space charge sign was re-inverted from negative to positive at about $150 \mathrm{~K}$. For electron trapping, however, the space charge went more negative at about $155 \mathrm{~K}$. The three transition temperatures associated to the three steps in space charge transformations are identified to be related to three deep levels: two for hole trapping, and one for electron trapping [24]. In an ideal situation, one may manipulate the space charge concentration in such a way that its concentration is minimum, thus greatly reducing the sensor full depletion voltage. At a given voltage, the reduction in space charge concentration results in the increase of the depletion depth, leading to an increase in the sensor CCE. In fact, late measurements on sensor CCE of charges created by laser have shown significant increase in sensor CCE with trapping of free holes at cryogenic temperatures [13]. We note here that free carrier injection can be produced by laser, current (injection current, thermal generation current, current caused by passing particles, etc). Figure 11 shows that, at a given cryogenic temperature, an optimal electric field distribution can be achieved in a Si sensor by adjusting the injection current density to an optimal value, typically in the order of 10's of nA [25]. However, with large enough injected carrier concentration/current, one can even achieve minimum/optimal space charge concentration even at or near RT [26].

The so-called Lazarus effect, the recovery of CCE in heavily irradiated Si sensors at cryogenic 


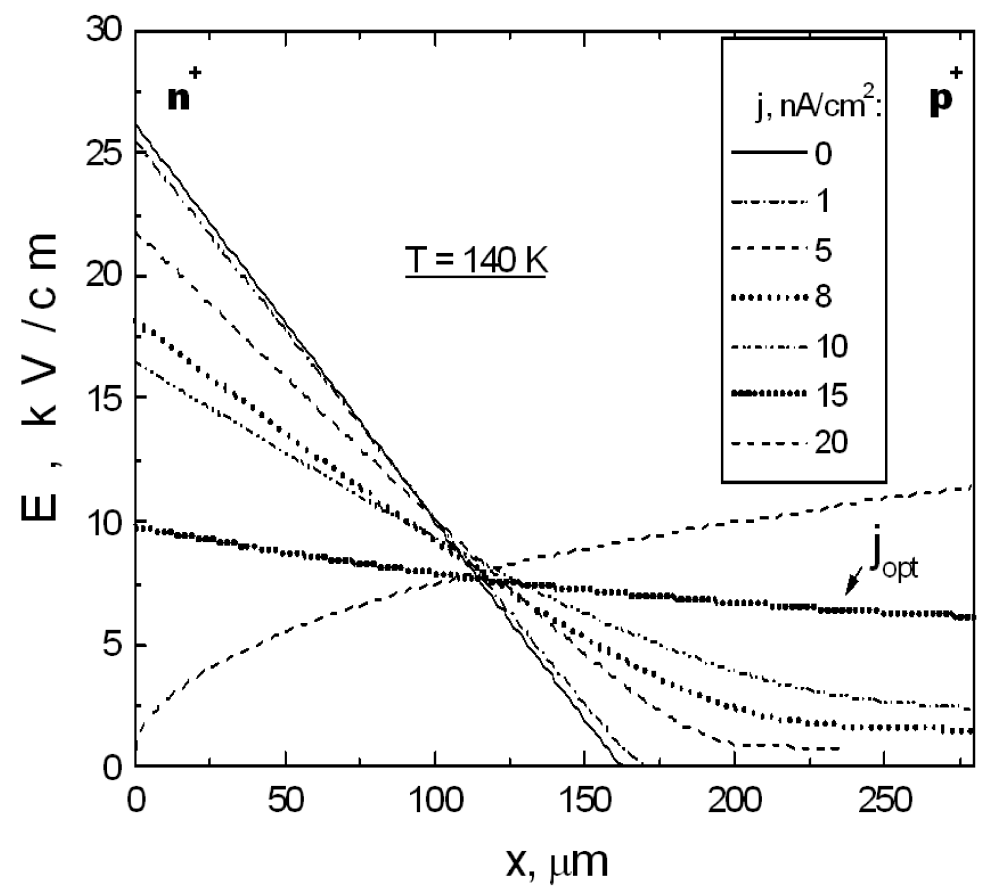

Figure 11. Simulated electric field distribution in irradiated Si sensor at various current injection conditions.

temperatures, have been discovered in 1998 [27], and it has been one of the main subjects for the CERN RD39 Collaboration [28]. Readers who are interested in more details of the Lazarus effect and radiation hard cryogenic Si detectors are referred to ref. [27-30].

\subsubsection{Double junction/double peak electric field distribution}

In 1992, we have observed in heavily irradiated $\mathrm{p}^{+} / \mathrm{n} / \mathrm{n}^{+}$Si detectors an abnormal electric field distribution deviating from its normal linear form. It has been found that, after being irradiated much beyond the SCSI, alpha particle induced current pulses can be seen from both sides of the detector that is not fully depleted, implying existence of a junction on either side of the detector [31]. This effect is also called the double junction (DJ) effect. A qualitative model with two deep levels, one donor level for the positive space charge near the $\mathrm{p}^{+}$contact, and one acceptor level for the negative space charge near the $\mathrm{n}^{+}$contact was proposed in ref. [31], as shown in figure 12. This abnormal electric field distribution in heavily irradiated Si sensors has been confirmed later in fully depleted sensor, where double peaks (DP) in current pulses induced by red lasers have been clearly seen [32, 33]. Figure 13 [33] shows the current pulse shapes induced by red laser illuminating on the $\mathrm{p}^{+}$contact, which produces electron current drifting from the $\mathrm{p}^{+}$contact to the $\mathrm{n}^{+}$contact. Since the current is nearly proportional to the electric field, a DP distribution in current indicates the same in the electric field distribution. 


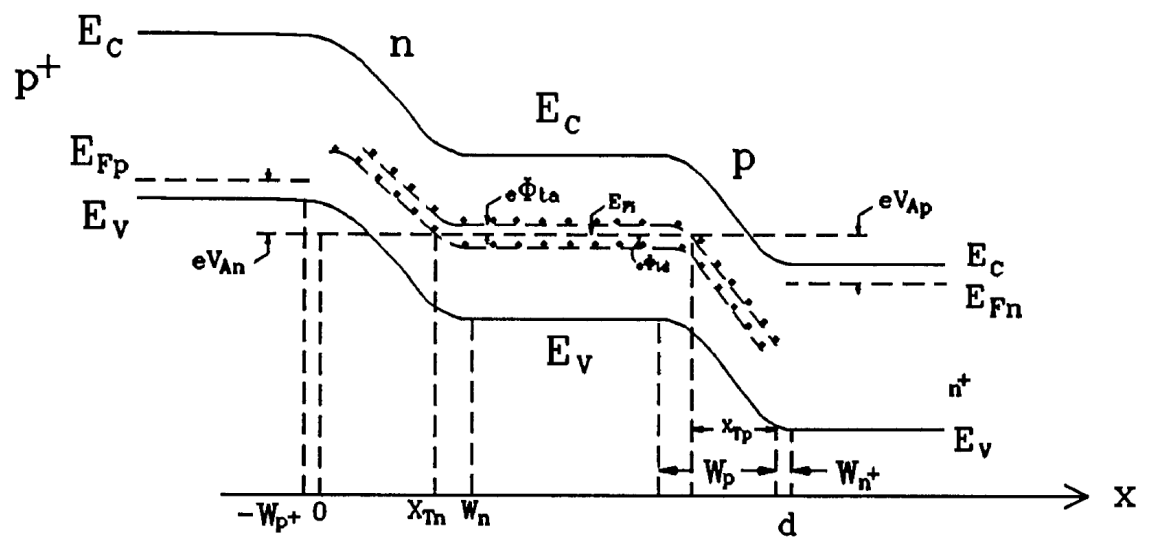

Figure 12. Energy band diagram for the proposed two-level model for the double junction effect.

\section{Radiation hardness of Si sensors - Previous approaches for LHC}

\subsection{Material/Impurity/Defect Engineering (MIDE)}

Material can be made more radiation hard by introduction of known impurities in the material purposefully. The known impurity introduced into the material, under the condition it will not significantly alter the material properties, can interact with the primary defects caused by radiation, vacancies and interstitials, to form such defect complexes that are much less damaging than the more intrinsic defects ( $\mathrm{V}-\mathrm{V}$, high order $\mathrm{V}$ complexes, and defect clusters). The role of theses known defects is to getter the radiation-induced primary defects. Detail modeling of defect kinetics has shown effectiveness of surplus oxygen in Si detectors in suppressing the formation of deep $\mathrm{V}-\mathrm{V}$ and $\mathrm{V}_{2} \mathrm{O}$ detects [34-36].

In 1992, BNL has developed a safe and reliable way of introducing oxygen into Si during the oxidation process [37]. The process, named High-temperature, long-time (HTLT) oxidation, is a small alternation from the conventional processing of high resistivity Si detectors. In the HTLT oxidation process, Si wafers are oxidized at $1150{ }^{\circ} \mathrm{C}$ to $1200{ }^{\circ} \mathrm{C}$ in an oxygen ambient for over 24 hours, resulting in an oxygen concentration over a few times of $10^{17} / \mathrm{cm}^{3}$ uniform over $50 \mu \mathrm{m}$ depth from either side of wafer. However, test results on these HTLT Si detectors to fast neutron radiation had shown no improved radiation hardness as compared to standard Si detectors [18].

In the time period of 1999-2000, in the interest of developing radiation hard Si detectors for LHC, where charged particles (protons, pions, etc) dominate, renewed interest had been generated to develop oxygenated Si detectors: 1) under the frame work of CERN RD48 Collaboration, a technology called Diffused Oxygen Float Zone Si (DOFZ Si) was developed, in which Si wafers were oxidized at high temperature $\left(1150{ }^{\circ} \mathrm{C}\right)$ for short period of time (a few hours) then drive-in (via diffusion) the oxygen in to the Si bulk in a $\mathrm{N}_{2}$ ambient at the same high temperature [38-41]; and 2) BNL had advanced its HTLT technology with even longer oxidation time ( 9 days) at $1200{ }^{\circ} \mathrm{C}$ to obtain uniform oxygen concentration of about $4 \times 10^{17} / \mathrm{cm}^{3}$ over the entire 300-400 $\mu \mathrm{m}$ thickness, with and without thermal donor (TD) [42]. Although again no radiation hardness to neutron radiation was observed, oxygenated Si detectors by either DOFZ or HTLT technology have been found more radiation hard to charged 


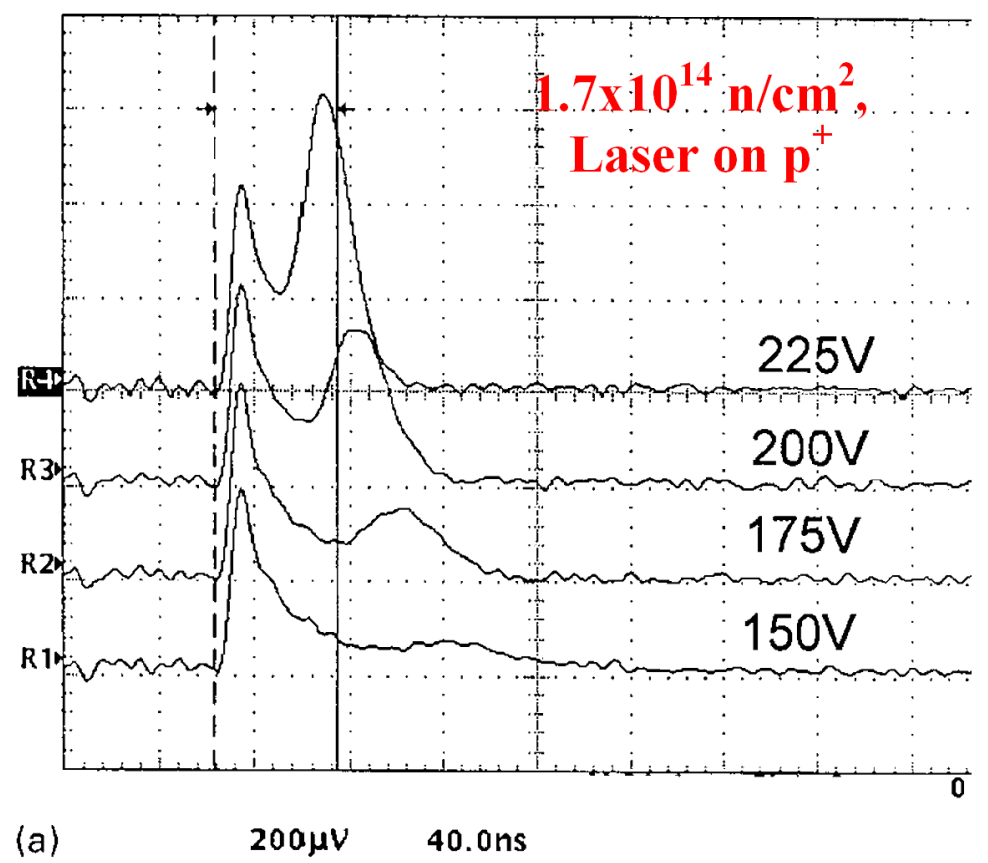

Figure 13. Red laser induced electron current shapes for irradiated Si sensors biased at various voltages. Double peaks in current shapes are clearly seen.

particle radiation (proton and pion) [38-42]. Figure $14[38,42]$ shows that, as compared to standard $\mathrm{Si}$ detector, the beta ( $\beta$ see eq. (2.2)) value is reduced by more than a factor of 2 in oxygenated $\mathrm{Si}$ detectors. Note that, for HTLT(TD) Si detectors, there is a wide flat range for beta (beta zero region) due to the fact that TD's are not removed by radiation. More interestingly, it has been found that oxygenated Si detectors are almost insensitive to gamma radiation up to $600 \mathrm{Mrads}$, as shown in figure 15a [42]. While standard Si detectors have gone through the normal SCSI due to negative space charge build up caused by gamma radiation, the full depletion voltage of oxygenated Si detectors has hardly changed. In fact, most resent data has shown that (Fig 15b, ref [43]), up to a dose of close to 2 Grad, there is a monotonical small build up of positive space charge in oxygenated detectors [43, 44].

A model has been proposed by BNL (BNL Model) that explains the difference in oxygen effect to different radiation source [45]. As shown in figure 16, and explained in section II, for neutron radiation, there are mostly defect clusters where the local ratio of $[\mathrm{O}] /[\mathrm{V}]$ can be very small $(\ll 0.1)$, preventing an effective gettering of V's by oxygen; while for gamma radiation, there are mostly point defects that are uniformly distributed in $\mathrm{Si}$, the ratio of $[\mathrm{O}] /[\mathrm{V}] \gg 1$, and the oxygen gettering effect is maximum. For charged particles, it is somewhere in between, and partial improvement of radiation hardness is observed. Simulations, done by Huhtinen [46], of defect formation in Si for various radiation sources has indeed shown that vacancies are mostly clustering in neutron irradiated $\mathrm{Si}$, for proton radiation, there are mixture of single vacancies and vacancy clusters. Recent DLTS results by Pintilie et al. have shown that, in standard Si detectors irradiated by gamma ray, there are almost one 


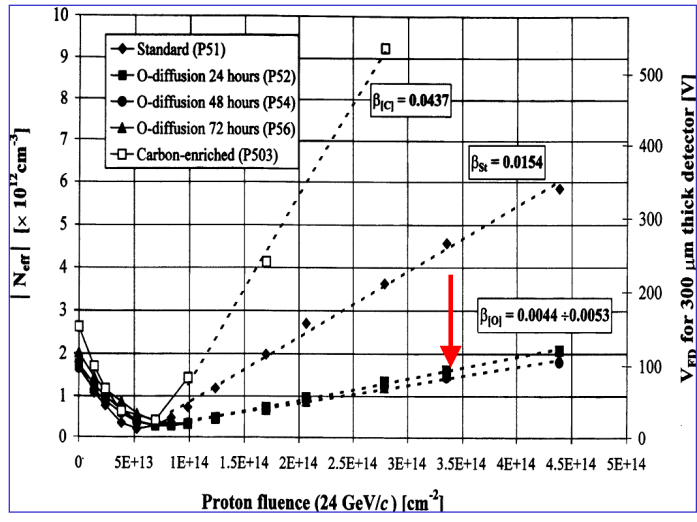

(a)

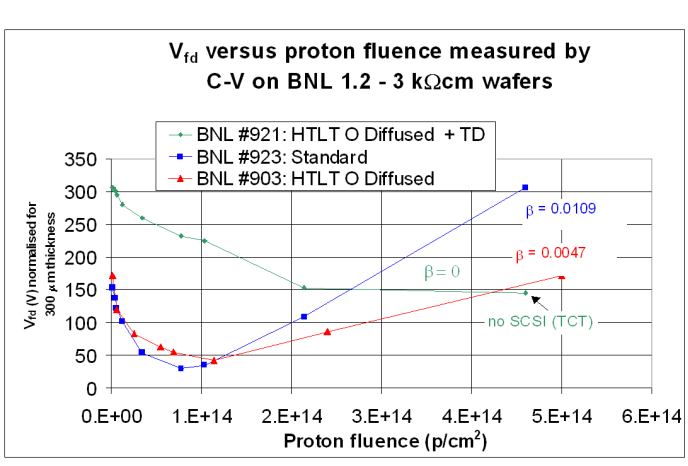

(b)

Figure 14. Changes in full depletion voltage and/or space charge concentration as a function of $24 \mathrm{GeV}$ proton fluence for oxygenated Si detectors as compared to standard Si detectors: a) HTLT and HTLT(TD) detectors; and b) DOFZ detectors.

to one correlation between measured concentration of deep level defects $\left(\mathrm{V}-\mathrm{V}, \mathrm{V}_{2} \mathrm{O}\right.$ or other V-related defects) and the detector leakage current and space charge concentration [47, 48]. In oxygenated $\mathrm{Si}$ detectors, both the leakage current and space charge concentration introduction rates were much lower, indicating that both $\mathrm{V}-\mathrm{V}$ and $\mathrm{V}_{2} \mathrm{O}$ deep level defects are greatly suppressed due to oxygen effect, in agreement with simulation results in ref $[35,36]$ and the proposed model.

Most recently, MCZ (Magnetic Czochralski) [49] and p-type Si detectors [50] are found more radiation hard in nature. For MCZ Si detectors, they are radiation hard due to their nature high oxygen concentration $\left(>4 \times 10^{17} / \mathrm{cm}^{3}\right)$, and for p-type Si detectors, it is due to the factor that they are non-inverting and $\mathrm{n}^{+}$electrodes collect electrons that are drifting much faster than holes in the electric field.

\subsection{Device Structure Engineering (DSE)}

As explained in section II, the main electrical degradation that affects Si detector operation most is the increase in detector full depletion voltage due to the negative space charge build up during radiation and during the RT storage (annealing). Major efforts have been made to improve the detector radiation tolerance by 1) increase the detector breakdown voltage using multi-guard rings; 2) decrease the detector full depletion voltage using novel detector structures (3D and Semi-3D detectors); and 3) decrease the detector full depletion voltage using thinner detectors.

\subsubsection{Multi-guard ring system}

Use of multi-guard ring system can redistribute the electric field over a larger distance along the detector edge, thus preventing break down along the detector edge at large bias voltages. The detector is thus operated at much larger biases than the initial full depletion voltage, and can tolerate larger radiation fluence before the detector is forced into partial depletion by radiation-induced negative space charge build up [51-53]. Figure 17 shows the breakdown characteristics of $n^{+} / p^{2} / p^{+}$Si detectors 


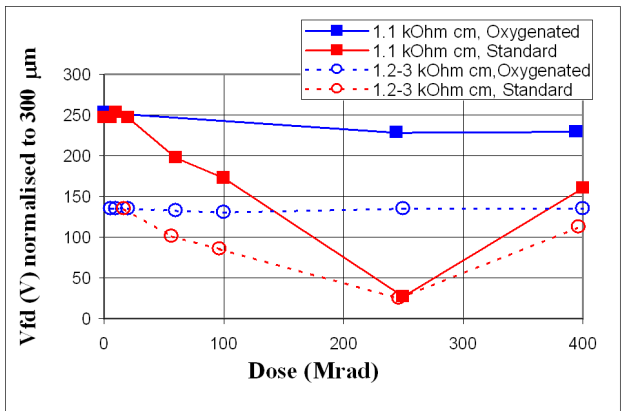

a)

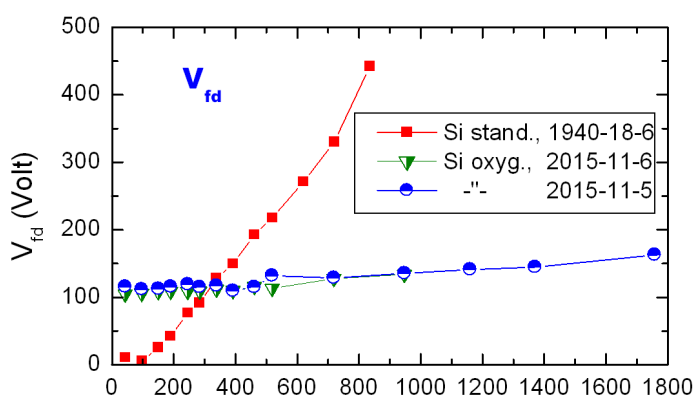

b)

Figure 15. Changes in full depletion voltage as a function of ${ }^{60} \mathrm{Co}$ gamma dose for HTLT oxygenated $\mathrm{Si}$ detectors as compared to standard Si detectors: a) early results at low doses; and b) recent results at high doses close to 2 Grad.

with different number of guard rings [53]. The initial full depletion voltage is about 100 volts. It is clear that, with 7 or more guard rings, the breakdown voltage can be pushed close to 1000 volts, for which an equivalent fluence of $6 \times 10^{14} \mathrm{n} / \mathrm{cm}^{2}$ can be tolerated.

In almost all experiments at LHC, multi-guard ring system has been used for Si strip and pixel detectors. Some even adapted the $\mathrm{n}^{+} / \mathrm{n} / \mathrm{p}^{+}$structure that has segmented electrodes on the $\mathrm{n}^{+}$side. In this case, after SCSI when the junction is shifted to the $\mathrm{n}^{+}$side, the detector may be still operational at partial depletion mode at very high radiation fluences.

\subsubsection{Semi-3D detectors}

Stimulated by the lateral depletion nature of the 3D detectors $[5,54]$, the novel idea of Semi-3D detectors was first developed by BNL in 2000 [55]. As shown in figure 18 for a $\mathrm{p}^{+}-\mathrm{n}^{+} / \mathrm{n}^{-} \mathrm{n}^{+}$Semi-3D Si strip detector, both $\mathrm{p}^{+}$and $\mathrm{n}^{+}$strips are implemented on the front side. The backside is again a uniform $\mathrm{n}^{+}$implant. In this novel structure, all the $\mathrm{n}^{+}$strips are tied up together to a positive bias that can be smaller or equal to the positive bias on the back $\mathrm{n}^{+}$plane, depending on the application needs. Each $\mathrm{p}^{+}$strip is connected to an electronics channel for signal readout. Before SCSI, the depletion goes both vertically from $\mathrm{p}^{+}$strips to the back $\mathrm{n}^{+}$plane, as in the conventional single-sided strip detectors, and laterally from $\mathrm{p}^{+}$strips to the neighboring $\mathrm{n}^{+}$strips. Since the depletion, originated from $\mathrm{p}^{+}$strips, has to go both vertically and laterally before SCSI, the full depletion voltage will be higher than a conventional single-sided $\mathrm{p}^{+} / \mathrm{n} / \mathrm{n}^{+}$strip detector, typically by $20 \%$, as predicted by simulations. As the radiation goes, before the SCSI, the overall full depletion will go down due to the combined effect of donor removal and acceptor creation, as discussed in previous section. The nature of depletion still stays the same. However, when the radiation reaches the point beyond the SCSI, there is a dramatic change in the nature of depletion: the depletion now originates from both the $\mathrm{n}^{+}$ strips in the front side, and the $\mathrm{n}^{+}$plane from the backside to the $\mathrm{p}^{+}$readout electrodes now being the Ohmic strips. The detector is basically depleted from both sides, thus dramatically decreases the full depletion voltage. In theory, this reduction in full depletion voltage for the same thickness, as compare to the one-sided depletion in the case of conventional single or double-sided Si strip detectors, should 
Model for the role of oxygen in rad-hardness

\begin{tabular}{|c|c|c|c|}
\hline $\begin{array}{l}\text { Particle } \\
\text { type }\end{array}$ & $\begin{array}{l}\text { Single } \\
\text { defects }\end{array}$ & $\begin{array}{l}\text { Defect } \\
\text { clusters }\end{array}$ & $\begin{array}{l}\text { Oxygen } \\
\text { effect }\end{array}$ \\
\hline n & $\mathbf{x}$ & $\begin{array}{l}\mathbf{x x x x x x} \\
\mathbf{R}_{\mathrm{V}-\mathrm{V}}>>1 \\
\end{array}$ & No \\
\hline $\begin{array}{l}\text { Charged } \\
\text { particles } \\
(p, \pi \text {, etc.) }\end{array}$ & $\begin{array}{l}\mathbf{x x x x} \\
\mathbf{R}_{\mathrm{V}-\mathrm{V}}<<1\end{array}$ & $\mathbf{x x}$ & Partial \\
\hline$\gamma$ & $\begin{array}{l}\mathbf{x x x x x x} \\
\mathbf{R}_{\mathrm{V}-\mathrm{V}}<<\mathbf{1}\end{array}$ & & Yes \\
\hline
\end{tabular}
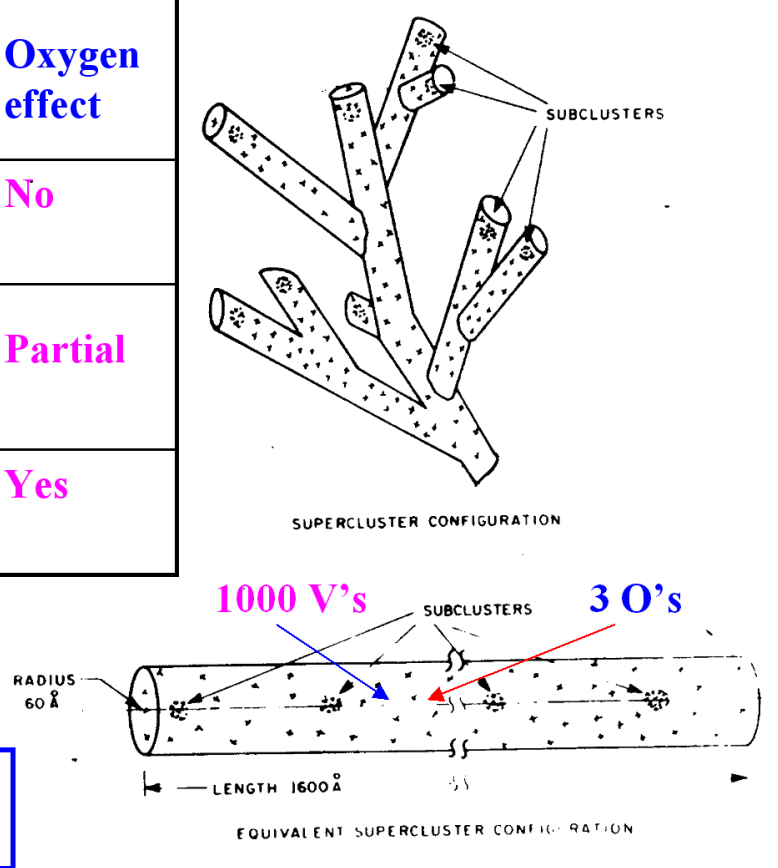

Z. Li et al., Nucl. Inst. \& Meth., A461 (2001) 126-132

Figure 16. The BNL model for the difference in oxygen effects to different types of radiations.

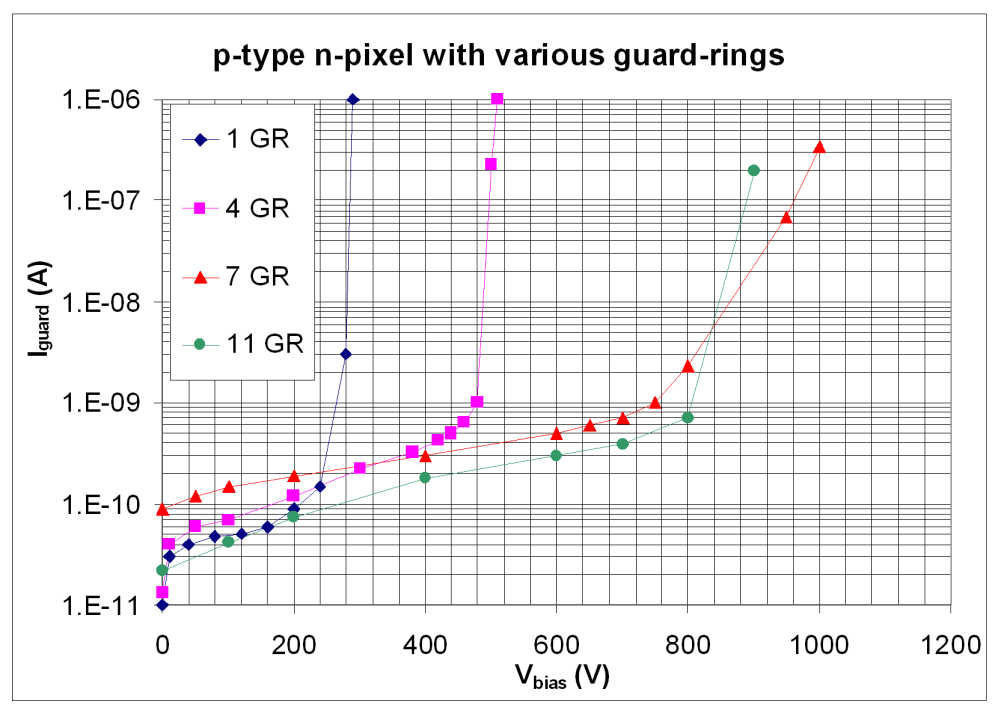

Figure 17. Current breakdown characteristics for $\mathrm{n}^{+} / \mathrm{p} / \mathrm{p}^{+}$detectors with various guard ring systems. 


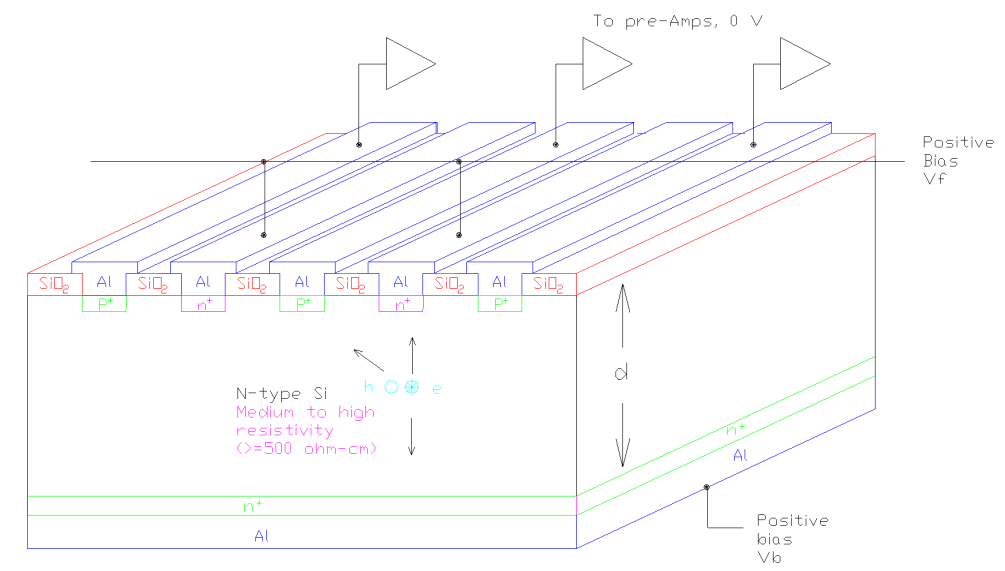

Figure 18. Schematics of a novel $\mathrm{p}^{+}-\mathrm{n}^{+} / \mathrm{n} / \mathrm{n}^{+}$Semi-3D Si strip detector.

be a factor of 4. However, as shown in ref. [56] in test results, due to the fact that the front $\mathrm{n}^{+}$ strips also originate the depletion laterally to the neighboring $\mathrm{p}^{+}$strips, which slows down the vertical depletion, the actual reduction factor is about 3.

\subsubsection{D detectors}

Developed by Park at University of Hawaii [54], the 3D detector got its name from the way it is processed. Different from conventional planar technology, $\mathrm{p}^{+}$and $\mathrm{n}^{+}$electrodes are diffused in small holes along the detector thickness ("3-d" processing), as shown in figure 19. The depletion develops laterally between the $\mathrm{p}^{+}$and $\mathrm{n}^{+}$electrodes that can be made 50 to $100 \mu \mathrm{m}$ in separation. The detector full depletion voltage thus just depends on the $\mathrm{p}^{+}-\mathrm{n}^{+}$separation, and it is insensitive to detector thickness. Since this separation can be made very small $(<100 \mu \mathrm{m})$, much less voltage will be used to fully deplete the detector, therefore providing much higher radiation tolerance at much lower biases. figure 20 shows that the $3 \mathrm{D}$ detector can be fully depleted at about 100 volts even after $1 \times 10^{15}$ $\mathrm{p} / \mathrm{cm}^{2} 55 \mathrm{MeV}$ proton radiation, which provides a factor of 8-10 in full depletion voltage reduction as compare to conventional planar Si detectors. We will discuss more about 3D Si detectors for SLHC in the next section.

\subsubsection{Thin detectors}

One obvious and easy way to reduce the detector full depletion voltage is to reduce the detector thickness. Compare to a Si detector with standard thickness of $300 \mu \mathrm{m}$, a thin detector with a thickness of $50 \mu \mathrm{m}$ can be fully depleted by a bias that is 36 times smaller, a reduction proportional to the square of the detector thickness. The drawbacks for thin detectors are: 1) signal to MIP will be reduced as well, a reduction proportional to the detector thickness; 2) increase in detector capacitance, leading to increase in noise; and 3) decrease in signal to noise ratio. However, in some applications when detectors with extremely high radiation tolerance, fast response, and/or extremely low mass are required, thin detectors can be one of the natural choices. 


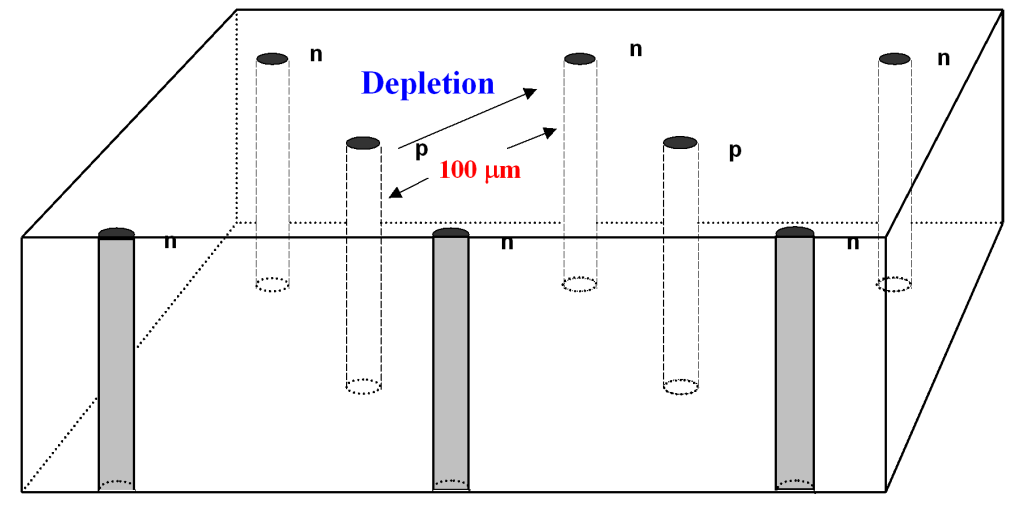

Figure 19. Schematics of a 3D Si detector.

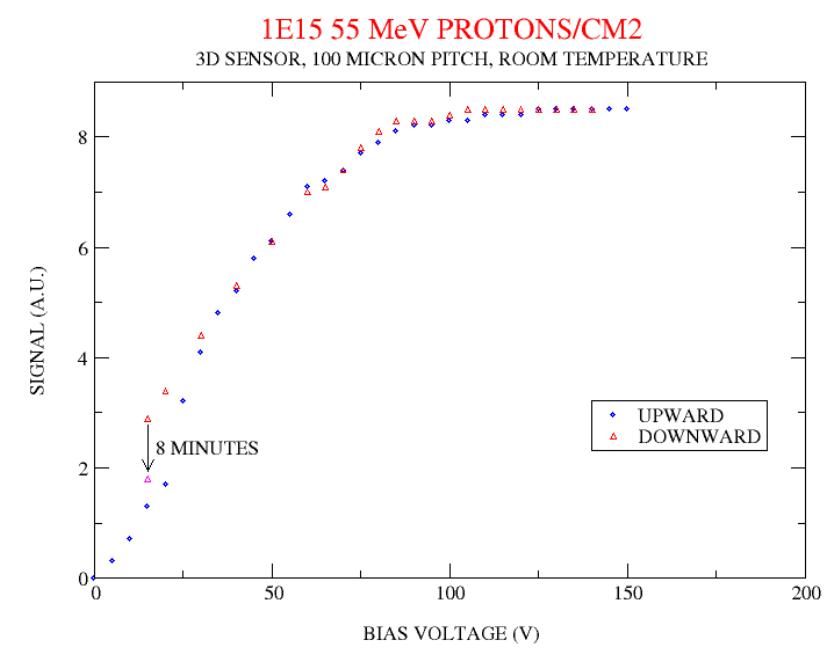

Figure 20. CCE of a 3D Si detector irradiated by $55 \mathrm{MeV}$ protons up to $1 \times 10^{15} \mathrm{p} / \mathrm{cm}^{2}$.

Thin detectors are more radiation tolerance. For $\mathrm{d}=50 \mu \mathrm{m}$, the detector can be still fully depleted up to a fluence of $2-3 \times 10^{15} \mathrm{n} / \mathrm{cm}^{2}$ at a bias of $200 \mathrm{~V}$ : 1) for a low starting resistivity Si $(50 \Omega-\mathrm{cm}$ ) detectors, there will be no SCSI up to $1.5 \times 10^{15} \mathrm{n} / \mathrm{cm}^{2}$; and 2) for high starting resistivity $\mathrm{Si}(4 \mathrm{k} \Omega-\mathrm{cm})$ detectors, they can be still fully depleted up to $3 \times 10^{15} \mathrm{n} / \mathrm{cm}^{2}$, even though SCSI taking place at about $1 \times 10^{13} \mathrm{n} / \mathrm{cm}^{2}$.

\section{Possible solutions for radiation tolerant Si detectors for SLHC}

\subsection{D detectors in various configurations}

As we discussed in the Introduction section, 3D Si detectors offer a scheme to decouple the detector depletion width from the free carrier drift length. As a result, according to simulation (see eq. (2.1)), the collected charge in a 3D detector can be amplified by a factor of $d / \lambda_{C}$ as compared to standard 


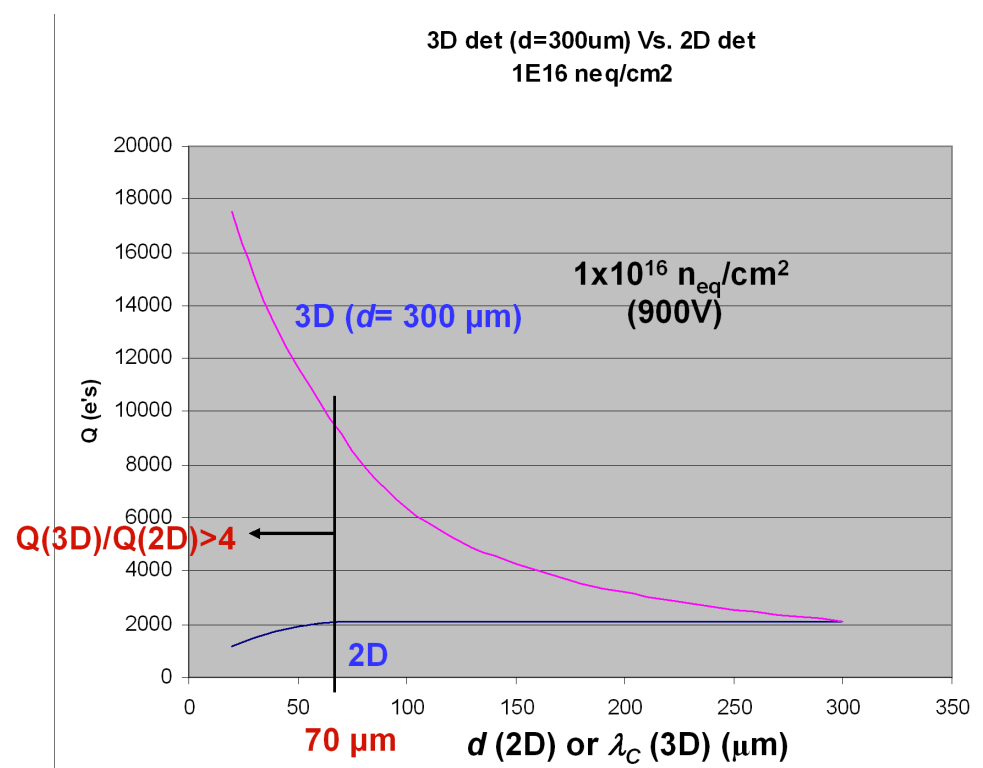

Figure 21. Calculated collected charge for 3D Si detectors with various column spacing as compared to that for 2D Si detectors with various thickness.

planar detectors (2D detectors), which can be as higher as 6 in a typical 3D detector with $50 \mu \mathrm{m}$ column spacing $\left(\lambda_{C}\right)$ and $300 \mu \mathrm{m}$ in detector thickness $(d)$. Figure 21 shows the calculated collected charge for 3D Si detectors with various column spacing as compared to that for 2D Si detectors with various thickness after being irradiated to $1 \times 10^{16} \mathrm{n}_{\mathrm{eq}} / \mathrm{cm}^{2}$. The calculation has been dome for MIP induced charges with both electron and hole contribution, resulting in a higher collected charge than that predicted by the calculation in section I. The amplification effect of collected charge in 3D detectors as compared to the 2D planar detectors can be greater than 4 with column spacing less or equal to $70 \mu \mathrm{m}$. It is clear that the 3D Si detectors are therefore one of the attractive choices for radiation hard/tolerant detectors for SLHC, where the radiation level in 10 years can be as high as $1 \times 10^{16} \mathrm{n}^{\mathrm{eq}} / \mathrm{cm}^{2}$. In the last 4 years, much progress has been made on optimizing the standard 3D Si detectors for higher charge collection and on developing new types of 3D Si detectors for better/easier processing and operation.

Figure 22 shows the most recent results of charge collection in the standard 3D Si detectors with various column spacing and irradiation to different fluences [57]. As predicted by eq. (2.1) and results in figure 21, the collected charge and charge collection efficiency increase with reduced column spacing. The experimental result of 11,000 electrons collected by a 3D detector with $70 \mu \mathrm{m}$ spacing (3E detector) at $8.8 \times 10^{15} \mathrm{n}_{\mathrm{eq}} / \mathrm{cm}^{2}$ fluence, which is about 9,600 electrons when normalized to $1 \times 10^{16} \mathrm{n}_{\mathrm{eq}} / \mathrm{cm}^{2}$, is very close to our calculated result of 9,500 electrons. In fact as shown in Table III, our calculated results fit very well with the experimental results for all three types of 3D detector with different column spacing shown in figure 22a. As shown in figure 22b, the charge collection efficiency at $8.8 \times 10^{15} \mathrm{n}_{\mathrm{eq}} / \mathrm{cm}^{2}$ increases from $40 \%$ to $65 \%$ when the column spacing is reduced from $107 \mu \mathrm{m}$ to $55 \mu \mathrm{m}$ [57]. 


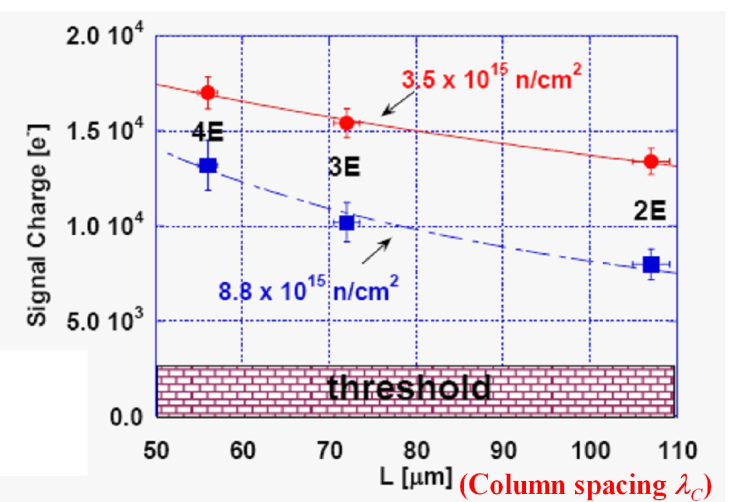

(a)

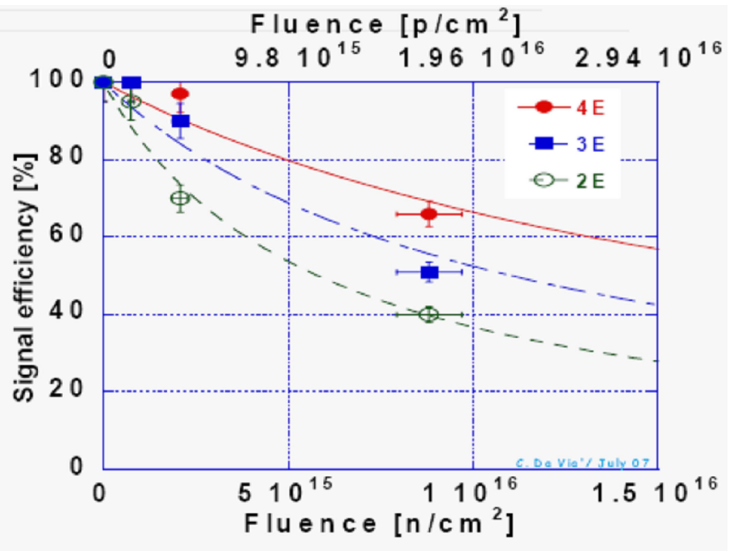

(b)

Figure 22. Latest charge collection results for 3D Si detectors a) as a function of column spacing; and b) as a function of radiation fluence [57].

Table 3. Comparisons of collected charge between calculation and measurement for 3D detectors with various column spacing. Collected charge is normalized to $1 \times 10^{16} \mathrm{n}_{\mathrm{eq}} / \mathrm{cm}^{2}$.

\begin{tabular}{|c|c|c|c|}
\hline Column spacing $\left(\lambda_{C} \quad \mu \mathrm{m}\right)$ & 107 & 70 & 55 \\
\hline$Q$ (e's) Calculation & 6300 & 9500 & 11200 \\
\hline$Q$ (e's) Measurement [57] & 6600 & 9600 & 11100 \\
\hline
\end{tabular}

In recent years, lots of efforts have been directed towards the development of new types of 3D Si detectors to simplify the fabrication process and/or read out scheme. Chief among them are: 1) the first single type column 3D (STC-3D) Si detectors, in which only one type of electrode columns (junctions) are etched from one side (not all the way through the detector thickness) while the back side is uniformly implanted to form the Ohmic contact [58]; 2) the first true single-sided processed 3D Si detectors, in which either two types of columns (junction and Ohmic) or single type columns (junction columns, the Ohmic electrodes are formed by ion implantation also done on the front side) are etched (not all the way through the detector thickness,) from the front side while the back side is left entirely un-touched [59]; and 3) the first true double-sided 3D Si detectors, in which the junction type columns are etched from the front side (not all the way through the detector thickness) and the Ohmic columns are etched from the back side (also not all the way through the detector thickness) and connected by uniform implants on the back side [60](see figure 23). While the process of the STC 3D Si detector (Type 1, and STC one in Type 2) is much easier as compared to the standard 3D Si detector process, the electric filed in these detectors are quite low, resulting in large drift time of carriers and therefore large trapping effect after heavy irradiation. The true-one sided dual-column 3D Si detectors (Type 2) offers much simpler process while preserve the radiation hard characteristics of standard 3D Si detectors. There is dead space in the last $20 \mu \mathrm{m}$ or so near the back side where the columns do not extend to, resulting in less than $7 \%$ in charge not collected. The true 2-sided 3D Si detectors (Type 


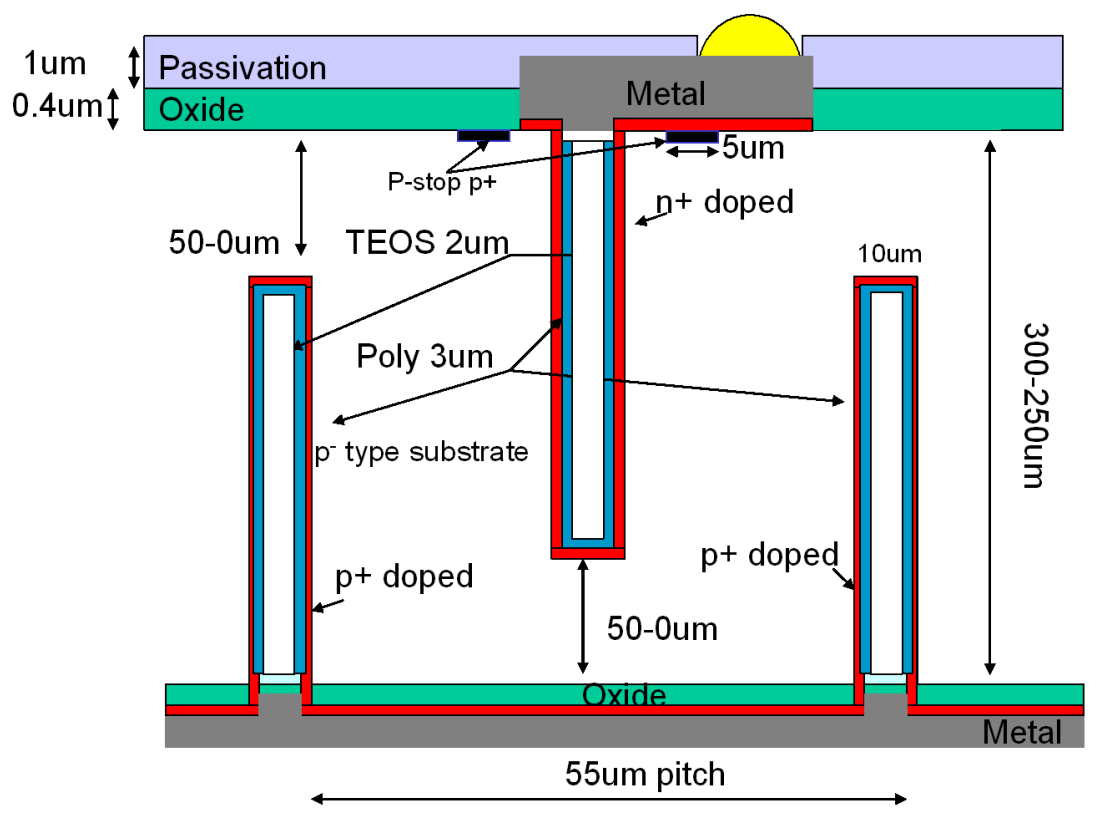

Figure 23. Schematic of the true two-sided dual-type column 3D Si detectors [60].

3) offer double-side accessibility, which in some cases are beneficial in terms of reducing the electric field along the surface and separating the electronics with different bias polarities on opposite sides.

\subsection{Current-Injected-Diode detectors}

Current-Injected-Diode (CID) concept has been developed by CERN RD39 collaboration [61]. It is based on the current injection to the detector sensitive volume. Figure 24 shows the operation principle of the CID in a space-charge-limited-current (SCLC) mode. The electric field is proportional to $\sqrt{x}$, with no explicit dependence on the radiation fluence. In this new approach the detector operates in the mode of "self-limited injected current", which maintains automatically a stable electric field distribution in the detector at any fluence.

The solution of the continuity and Poisson equations using the Shockley-Read-Hall statistics for the carriers trapped by deep levels gives the trapped charge density distribution. Figure 25 shows the calculated $E(x)$ for the $\mathrm{p}^{+}-\mathrm{n}-\mathrm{p}^{+}$structure with a deep level trapping center of the activation energy $\mathrm{E}_{t}-\mathrm{E}_{V}=0.48 \mathrm{eV}$ and the concentration of $N_{t} \approx 2.8 \times 10^{12} \mathrm{~cm}^{-3}$.

The important feature of $E(x)$ is that the electric field extends over the entire detector thickness, indicating a virtual full depletion. The electric field has a maximum at the contact opposite to the injecting contact, whose value depends only on the detector thickness $d$ and the bias voltage $V$. In the CID operation mode, the $E(x)$ profile is independent on the material properties and has no sensitivity to radiation influence.

The calculated $E(x)$ distribution at $\mathrm{V}=30 \mathrm{~V}$ (figure 3) well illustrates that the detector is fully depleted, whereas without injection the depth of the space charge region is $120 \mu \mathrm{m}$ only, which is limited by the concentration of electrically active deep levels of $2.8 \times 10^{12} \mathrm{~cm}^{-3}$. This effective trap concentration is resulted by the irradiation with approximately $5 \times 10^{14} \mathrm{n}_{\mathrm{eq}} / \mathrm{cm}^{2}$. 

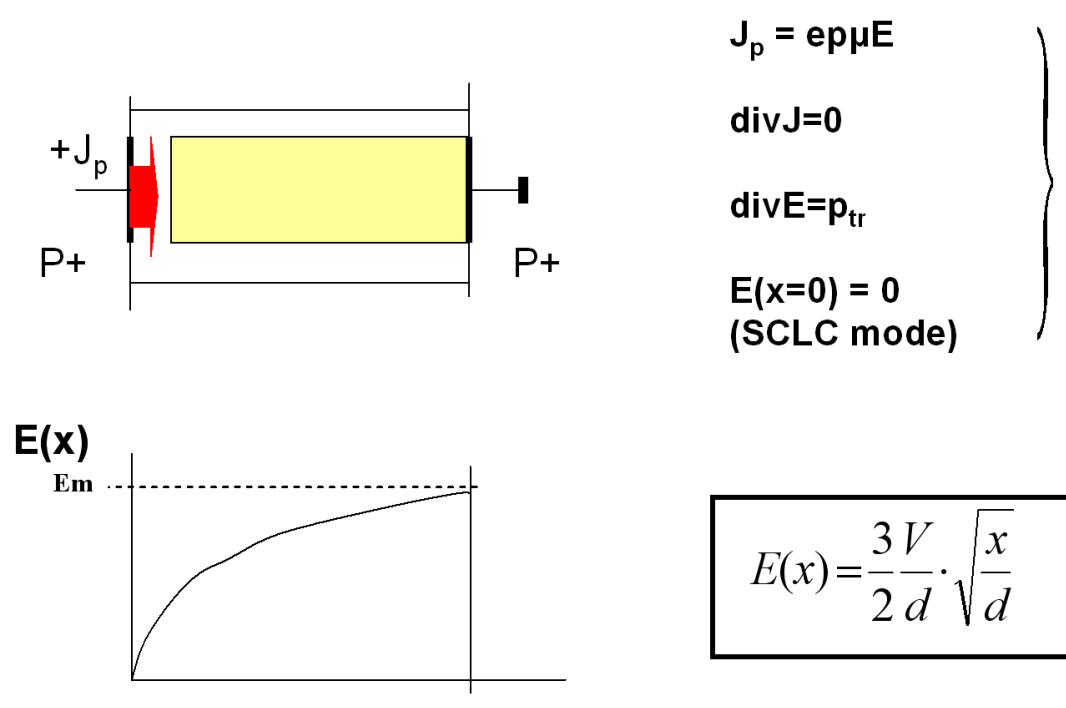

$$
E(x)=\frac{3}{2} \frac{V}{d} \cdot \sqrt{\frac{x}{d}} \quad E_{m}=\frac{3}{2} \cdot \frac{V}{d}
$$

Figure 24. The principle of the CID operation [61].

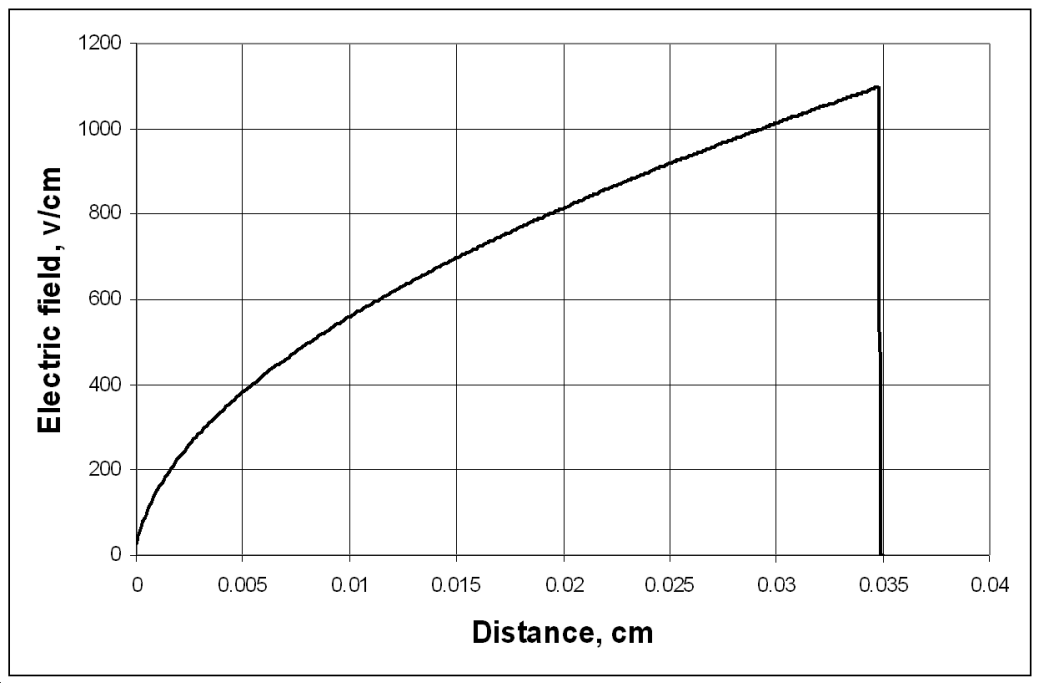

Figure 25. Electric field distribution calculated for CID with hole injection at: $\mathrm{J}_{\mathrm{inj}}=1 \times 10^{-5} \mathrm{~A} / \mathrm{cm}^{2}, \mathrm{~V}=30 \mathrm{~V}$, $\mathrm{N}_{d l}=2.8 \times 10^{12} \mathrm{~cm}^{-3}[61]$.

The I-V characteristic of a CID calculated with the same parameters as those for figure 25 is shown in figure 26. The SCLC mode defines specific current voltage (I-V) characteristic with three major features: the linear (ohmic) I-V dependence at low current, square I-V dependence at the SCLC mode for the major region of I-V curve, and a sharp current rise at a certain bias voltage. The Ohmic part corresponds to a low injected current density, which cannot disturb the free currier concentration in the detector bulk material. The quadratic part is a space charge limited current with the concentration of the injected curriers $n \gg n_{0}$. The sharp current rise is a result of the deep level trap saturation when all of the traps are completely filled. This saturation prevents future self-adjustment of the trapped charge density to the bias. In this case any increase of bias will lead to non-limited current 


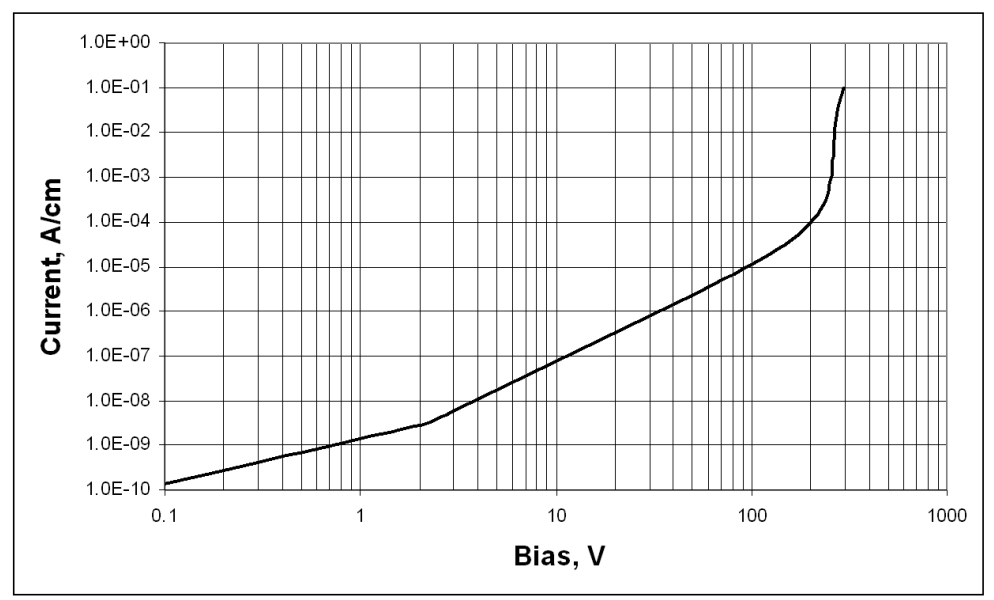

Figure 26. Calculated I-V characteristic for CID. Parameters for calculation are the same as in figure 25. [61].

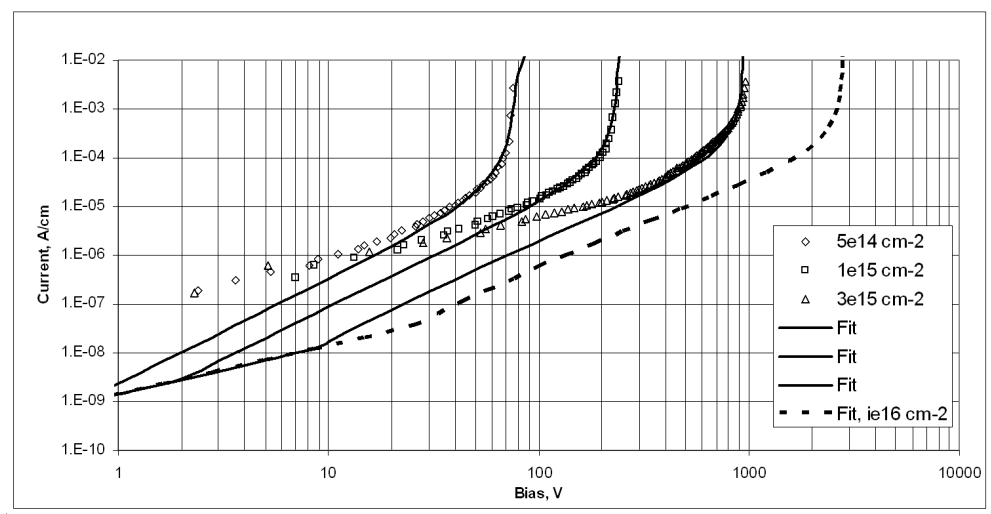

Figure 27. Experimental and simulated normalized I-V's of CID irradiated by different fluencies and operated at $220 \mathrm{~K}$ [61].

injection until the injected free carriers itself start to influence on the electric field distribution. This will require $n_{i} N_{t}$. It should be emphasized that the sharp rise of the current is not related to any breakdown effects.

One important point for CID application is the range of the operational bias and the value of operational or leakage current. Figure 27 presents the set of I-V's for CID irradiated by different neutron fluencies. The detectors were processed on n-type MCz-Si high resistivity silicon wafers with thickness of $300 \mu \mathrm{m}$. The experimental I-Vs were simulated with the same deep level trapping centers used above. The only parameter that was adjusted in the simulation is the concentration of trapping centers $N_{t}$.

The value of $N_{t}$ used for the fitting depends nearly linearly on fluence with a proportional coefficient of about $d N_{t} / d F=2.5 \times 10^{-2} \mathrm{~cm}^{-1}$. This parameterization allows one to calculate the I-V's at $\mathrm{F}>1 \times 10^{16} \mathrm{~cm}^{-2}$, which is presented in figure 27 as a dashed line.

The important advantage of CID is that at certain value of the bias the current reduces with fluence. An increase of fluence from $1 \times 10^{15}$ to $3 \times 10^{15} \mathrm{n}_{\mathrm{eq}} / \mathrm{cm}^{2}$ for a CID biased at $200 \mathrm{~V}$ leads to a 


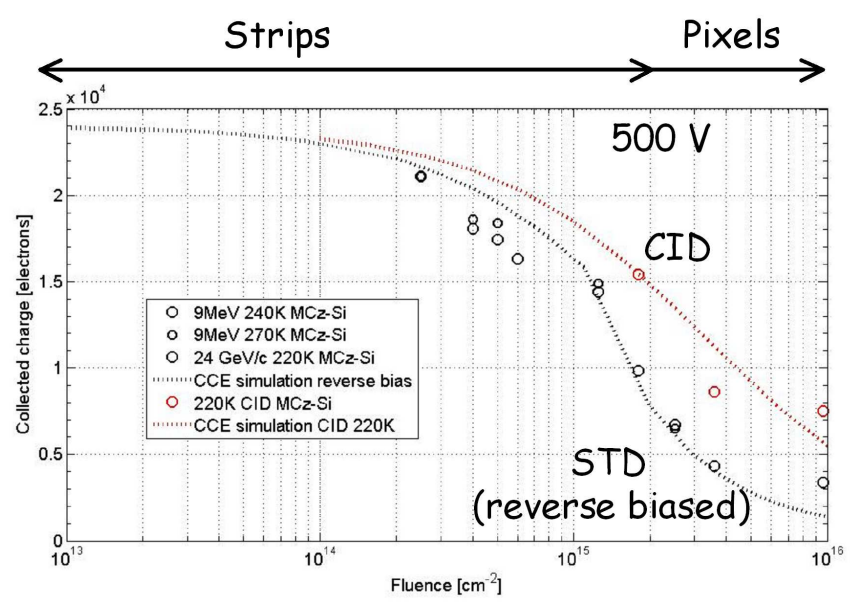

Figure 28. Experimental and simulated collected charges of CID and reverse-biased Si pad detectors irradiated by different fluencies [62].

current reduction of about 1 order of the magnitude. This is a unique feature of CID, which can be beneficial in SLHC applications.

Shown in figure 28 is the measured charge collection of irradiated CID pad detectors using a Cryogenic TCT with an infrared laser to simulate MIP [62]. It is clear that when detectors operated in a CID mode (forward biased in this case), the charge collection is much larger (about 3-times larger) than that in a standard mode (reverse biased). It is very important to note that, good fitting to the CID data can only be made by increasing the trapping time constant by a factor of 5-10, indicating much less trapping in the CID detector as well. The suppression of trapping in CID detectors can be explained by the filling of traps by injected current, which makes the traps occupied and therefore no longer active in trapping free carrier generated by particles to be detected. As many as 7000 electrons, which is close the number by a 3D Si detector (see table III), can be collected by CID detectors after $1 \times 10^{16} \mathrm{n}_{\mathrm{eq}} / \mathrm{cm}^{2}$ radiation at a modest temperature of $-53{ }^{\circ} \mathrm{C}$, which can be achieved by liquid $\mathrm{CO}_{2}$ cooling.

\subsection{Elevated temperature annealing}

The most obvious solution to deal with the radiation induced damage is to remove them by elevated temperature annealing. But this procedure has not been tried until two years ago, when BNL tried elevated temperature annealing termed as DRIVE (Device Recovery/Improvement Via Elevated temp annealing) [62]. The reason for this is that it had been feared that elevated temperature can cause reverse annealing as we discussed in previous section at temperatures lower than $400{ }^{\circ} \mathrm{C}$, or cause spiking in the junction and therefore high leakage current at temperatures higher than $450{ }^{\circ} \mathrm{C}$. In fact in the initial stages of the first try of the DRIVE approach, the reverse annealing was the main effect, as can be seen in figure 29, where absolute value of $N_{\text {eff }}$ of an irradiated MCZ Si detector was going up with annealing temperature lower than $400{ }^{\circ} \mathrm{C}$. Only extended annealing at higher temperatures (about $450{ }^{\circ} \mathrm{C}$ ) makes the absolute value of $N_{\text {eff }}$ decrease, and eventually change sign from negative to positive (space charge sign inversion again, this time is from negative back to positive). As shown 


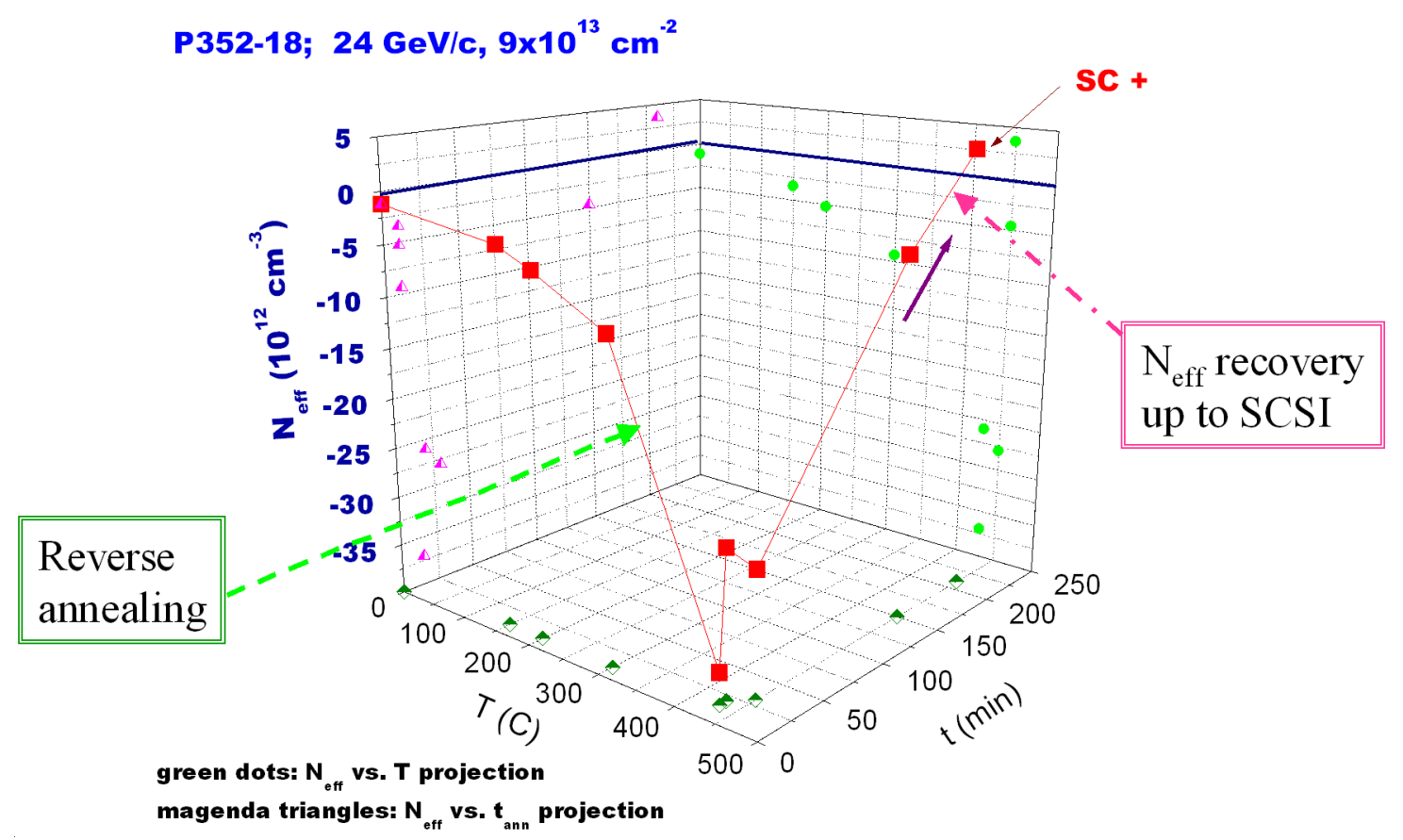

Figure 29. Evolution of space charge density with annealing temperature and time for an irradiated MCZ Si detector [62].

figure 30, the leakage current of an irradiated MCZ Si detector goes down with elevated temperature monotonically, which is very much beneficial to the detector operation. It is expect that defects related to trapping effect will also be annealed out by DRIVE, resulting in much improved charge collection.

There are many different ways to anneal detectors after being installed in the experiments [62]: 1) Annealing by self-heating generated by forward and/or reverse current; 2) annealing by laser or light; and 3) annealing by ultrasonic wave.

\subsection{Summary}

The details in displacement radiation damage in Si sensors have been reviewed in this paper. The main radiation induced damage in Si sensors and degradations in sensor performances can be summarized as the following:

1. The displacement radiation induced damage in Si is mainly in the form of deep level single defects and defect clusters (extended defect regions). For neutron radiation, it is mainly in the form of defect clusters. For gamma and electron radiations, it is mainly in the form of deep level single defects. For charge particles (protons, pions, etc.), it is the mixture of the two.

2. The first and most obvious radiation damage effect in Si sensor is the increase of sensor leakage current with radiation fluence. The cause for the leakage current increase is the thermal generation of carriers from radiation induced deep level defects and defect clusters. 


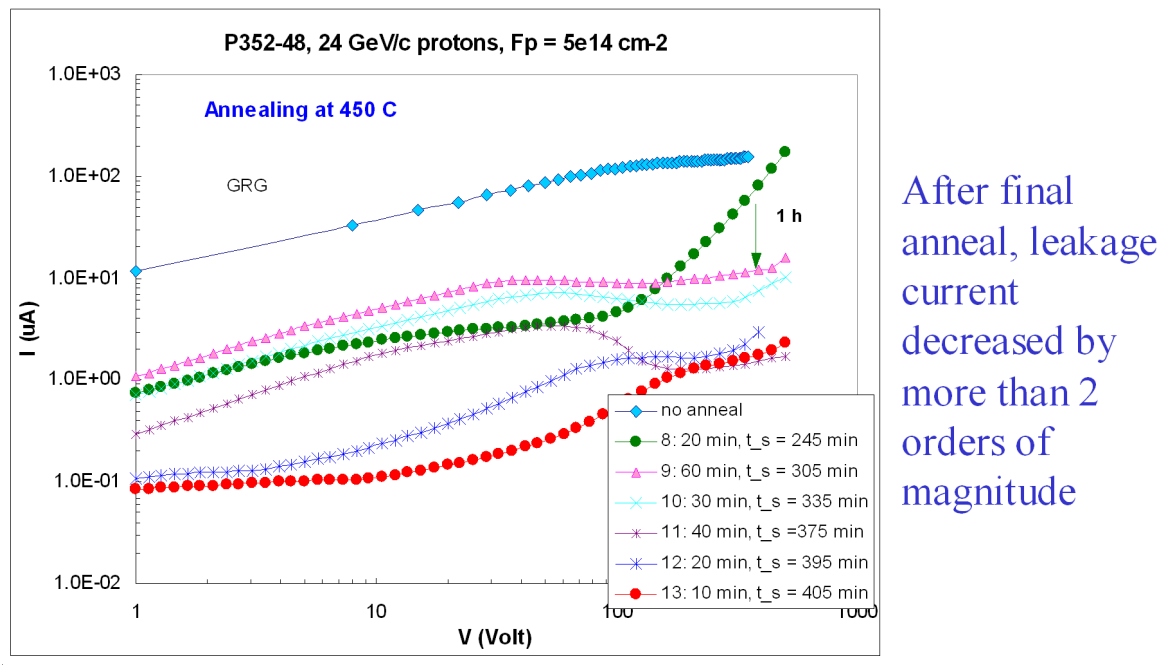

Figure 30. Evolution of leakage current with annealing temperature and time for an irradiated Si detector [62].

3. For the neutral bulk Si, the displacement damage is mainly reflected in the increase of bulk resistivity towards the intrinsic value ( $200 \mathrm{k}$ to $300 \mathrm{k} \Omega-\mathrm{cm}$ ) and decrease in carrier Hall mobility.

4. The space charge in the depletion region of Si sensors undergoes various transformations during radiation, anneal, and operation:

5. At very high fluences in SLHC environment $\left(4 \times 10^{15}\right.$ to $\left.1 \times 10^{16} \mathrm{n}_{\mathrm{eq}} / \mathrm{cm}^{2}\right)$, the main limiting factor for Si detector operation is free carrier trapping by radiation induced traps. At SLHC fluence, the charge collection distance can be only 20 to $50 \mu \mathrm{m}$, regardless of detector thickness and depletion depth.

Although radiation induced damage/degradation in $\mathrm{Si}$ sensors is quite extensive and severe, $\mathrm{Si}$ sensors can be, however, made more radiation hard/tolerant for LHC application by oxygenation (oxygenated $\mathrm{Si}$ and MCZ Si), by using multiple guard ring for high voltage operation; and by lower temperature operation. For SLHC application, however, 3D Si detector of various configurations, CID detector operation mode, and/or elevated temperature annealing may be necessary for much improved charge collection performance.

\section{Acknowledgments}

The author would like to thank our collaborators from Beijing Institute of Semiconductors, BNL (AGS, NSLS, Physics US ATLAS Upgrade), RHIC (PHENIX, STAR)), CERN (RD39, NA60, RD50); HIP, FNAL, JHU, Ioffe Physico-Technical Institute, LANL, Purdue University, UC Davis, University of Florence, University of Hamburg, University of Roma I, and Wayne State University for their contributions during various collaborating projects. This research was supported by the U.S. Department of Energy: contract No: DE-AC02-98ch10886, and was done partially within the framework of CERN RD50 and CERN RD39 Collaborations. 


\section{References}

[1] M. Bruzzi et al., Radiation-hard semiconductor detectors for SuperLHC, Nucl. Instrum. Meth. A541 (2005) 189.

[2] RD39 collaboration, E. Verbitskaya et al., The effect of charge collection recovery in silicon p-n junction detectors irradiated by different particles, Nucl. Instrum. Meth. A514 (2003) 47.

[3] E. Gatti, G. Padovini, and V. Radeka, Signal evaluation in multielectrode radiation detectors by means of a time dependent weighting vector, Nucl. Instrum. Meth. A 193 (1982) 651.

[4] V. Radeka, Low-noise techniques in detectors, Ann. Rev. Nucl. Part. Sci. 38 (1988) 217.

[5] S.I. Parker, C.J. Kenney and J. Segal, 3-D: a new architecture for solid state radiation detectors, Nucl. Instrum. Meth. A395 (1997) 328.

[6] Z. Li and H.W. Kraner, Modeling and simulation of charge collection properties for neutron irradiated silicon detectors, Nucl. Phys. B (Proc. Suppl.) 32 (1993) 398.

[7] H.W. Kraner, Z. Li and E. Fretwurst, The Use of the signal current pulse shape to study the internal electric field profile and trapping effects in neutron damaged silicon detectors, Nucl. Instrum. Meth. A326 (1993) 350.

[8] V.A.J. Van Lint, The physics of radiation damage in particle detectors, Nucl. Instrum. Meth. A253 (1987) 453.

[9] H.W. Kraner, Z. Li and K.U. Posnecker, Fast neutron damage in silicon detectors, Nucl. Instrum. Meth. A279 (1989) 266.

[10] Z. Li et al., Study of the long term stability of the effective concentration ofionized space charges $N_{\text {eff }}$ of neutron irradiated silicondetectors fabricated by various thermal oxidation processes, IEEE Trans. Nucl. Sci. 39 (1992) 1730.

[11] E. Verbitskaya et al., Competition of generation channels in reverse current of irradiated silicon detectors, presented at the $3^{\text {rd }}$ Conference on Radiation Effects on Semiconductor Materials, Detectors and Devices, June 28-30, Florence, Italy (2000).

[12] Z. Li et al., Effects of fast neutron radiation on the electrical properties of silicon detectors, Nucl. Instrum. Meth. A 308 (1991) 585.

[13] B. Dezillie et al., Improved neutron radiation hardness for Si detectors: Application of low resistivity starting material and/or manipulation of $N_{\mathrm{eff}}$ by selective filling of radiation-induced traps at low temperatures, IEEE Trans. Nucl. Sci. 46 (1999) 221.

[14] S. Pirollo et al., Radiation damage on p-type silicon detectors, Nucl. Instrum. Meth. A 426 (1999) 126.

[15] V. Eremin and Z. Li, Determination of the Fermi level position for neutron irradiated high resistivity silicon detectors and materials using the transient charge technique (TChT), IEEE Trans. Nucl. Sci. 41 (1994) 1907.

[16] R. Wunstorf, A systematic investigation of the radiation hardness of silicon detectors for high-energy physics experiments, Ph.D. Thesis, University od Hamburg, Hamburg, Germany (1992), DESY-FH1K-92-01.

[17] Z. Li and H.W. Kraner, Modeling and simulation of charge collection properties for neutron irradiated silicon detectors, Nucl. Phys. B 32 (1993) 398. 
[18] Z. Li et al., Study of the long term stability of the effective concentration of ionized space charges $\left(N_{\mathrm{eff}}\right)$ of neutron irradiated silicon detectors fabricated by various thermal oxidation processes, IEEE Trans. Nucl. Sci. 42 (1995) 219.

[19] Z. Li et al., Investigation on the $N_{\text {eff }}$ reverse annealing effect using TSC/I-DLTS: Relationship between neutron induced microscopic defects and silicon detector electrical degradations, Nucl. Instrum. Meth. A 377 (1996) 265.

[20] Z. Li, Experimental comparisons among various models for the reverse annealing of the effective concentration of ionized space charges (Neff) of neutron irradiated silicon detectors, IEEE Trans. Nucl. Sci. 42 (1995) 224.

[21] Z. Li et al., Study of bulk damage in high resistivity silicon detectors irradiated by high dose of ${ }^{60}$ Co-radiation, IEEE Trans. Nucl. Sci. NS-44 (1995) 834.

[22] V. Eremin, Z. Li and I. Ilyashenko, Trapping induced $N_{\mathrm{eff}}$ and electrical field transformation at different temperatures in neutron irradiated high resistivity silicon detectors, Nucl. Instrum. Meth. A360 (1995) 458.

[23] Z. Li et al., Direct observation and measurements of neutron-induced deep levels responsible for $N_{\mathrm{eff}}$ changes in high-resistivity silicon detectors using TCT, Nucl. Instrum. Meth. A 388 (1997) 297.

[24] E. Verbitskaya et al., Optimization of electric field distribution by free carrier injection in silicon detectors operated at low temperatures, IEEE Trans. Nucl. Sci. 49 (2002) 258.

[25] M. Zavrtanik et al., Temperature dependence of electron and hole signals in irradiated $p^{+}-n-n^{+}$ diodes in the presence of continuous carrier injection, IEEE Trans. Nucl. Sci. 49 (2002) 264.

[26] V. Palmieri, K. Borer, S. Janos, C. Da Via and L. Casagrande, Evidence for charge collection efficiency recovery in heavily irradiated silicon detectors operated at cryogenic temperatures, Nucl. Instrum. Meth. A413 (1998) 475.

[27] RD39 collaboration, K. Borer et al., Superconducting microstrip detectors: addendum to proposal DRDC-P-53, CERN/LHCC98-27.

[28] RD39 collaboration, L. Casagrande et al., Review on the development of cryogenic silicon detectors, Nucl. Instrum. Meth. A461 (2001) 150.

[29] RD39 collaboration, K. Borer et al., Charge collection efficiency of irradiated cryogenic double-p silicon detector, Nucl. Instrum. Meth. A462 (2001) 474.

[30] Z. Li and H.W. Kraner, Fast neutron radiation damage effects on high resistivity silicon junction detectors, J. Electron. Mat. 21 (1992) 701.

[31] V. Eremin, Z. Li and I. Ilyashenko, Trapping induced $N_{\mathrm{eff}}$ and electrical field transformation at different temperatures in neutron irradiated high resistivity silicon detectors, Nucl. Instrum. Meth. A360 (1995) 458.

[32] D. Menichelli et al., Modelling of observed double-junction effect, Nucl. Instrum. Meth. A 426 (1999) 135.

[33] V. Eremin, E. Verbitskaya and Z. Li, The origin of double peak electric field distribution in heavily irradiated silicon detectors, Nucl. Instrum. Meth. A476 (2002) 556.

[34] B. MacEvoy et al., Defect kinetics modelling; a brief review and some interesting new results, talk given at the $3^{\text {rd }}$ ROSE Workshop on Radiation Hardening of Silicon Detectors, February 12-14, Hamburg, 
Gemrany (1998).

[35] S. Lazanu et al., Microscopic modelling of defects production and their annealing after irradiation in silicon for HEP particle detectors, talk given at $4^{\text {th }}$ International Conference on Radiation Effects on Semiconductor Materials Detectors and Devices (RESMDD002), July 10-12, Florence, Italy (2002), Nucl. Instrum. Meth. A 514 (2003) 9.

[36] Z. Li et al., 1992 IEEE Nuclear and Space Radiation Effects Conference (NSREC'92), IEEE Trans. Nucl. Sci. 39 (1992) 1730.

[37] CERN RD48 collaboration, A Rizin et al., Recent results from the Rd48 (ROSE) collaboration, Nucl. Instrum. Meth. A 447 (2000) 116.

[38] G. Lindström et al., $3^{\text {rd }} R D 48$ status report: the ROSE collaboration ( $R \& D$ on silicon for future experiments), CERN-LHCC-2000-009.

[39] ROSE collaboration, G. Lindström et al., Radiation hard silicon detectors developments by the RD48 (ROSE) Collaboration, Nucl. Instrum. Meth. A466 (2001) 308.

[40] G. Lindström, Radiation damage in silicon detectors, talk given at $9^{\text {th }}$ European Symposium on Semiconductor Detectors, June 23-27, Schloss Elmau, Germany (2002), Nucl. Instrum. Meth. A 512 (2003) 30.

[41] B. Dezillie et al., The effect of oxygen impurities on radiation hardness of FZ silicon detectors for HEP after neutron, proton and gamma irradiation, IEEE Trans. Nucl. Sci. 47 (2000) 1892.

[42] Z. Li et al., Paradoxes of steady-state and pulse operational mode characteristics of silicon detectors irradiated by ultra-high doses of $\gamma$-rays, talk given at $4^{\text {th }}$ International Conference on Radiation Effects on Semiconductor Materials Detectors and Devices (RESMDD002), July 10-12, Florence, Italy (2002), Nucl. Instrum. Meth. A 514 (2003) 25.

[43] E. Fretwurst et al., Bulk damage effects in standard and oxygen-enriched silicon detectors induced by ${ }^{60}$ Co-gamma radiation, talk given at $4^{\text {th }}$ International Conference on Radiation Effects on Semiconductor Materials Detectors and Devices (RESMDD002), July 10-12, Florence, Italy (2002), Nucl. Instrum. Meth. A 514 (2003) 1.

[44] Z. Li et al., HTLT oxygenated silicon detectors: radiation hardness and long term stability, Nucl. Instrum. Meth. A461 (2001) 126.

[45] M. Huhtinen, Simulation of non-ionising energy loss and defect formation in silicon, Nucl. Instrum. Meth. A 491 (2003) 194 [ROSE/TN/2001-02].

[46] T. Pintilie et al., Close to midgap trapping level in ${ }^{60}$ Co gamma irradiated silicon detectors, Appl. Phys. Lett. 81 (2002) 165.

[47] I. Pintillie et al., Results on defects induced by ${ }^{60}$ Co gamma irradiation in standard and oxygen-enriched silicon, Nucl. Instrum. Meth. A514 (2003) 18.

[48] J. Härkönen et al., Proton irradiation results of $p^{+} / n^{-} / n^{+}$Cz-Si detectors processed on p-type boron-doped substrates with thermal donor-induced space charge sign inversion, Nucl. Instrum. Meth. A552 (2005) 43.

[49] G. Casse, Pixels, talk at the $3^{\text {rd }}$ Workshop on Advanced Silicon Radiation Detectors (3D and P-type Technologies), April 14-16, Barcelona, Spain (2008).

[50] Z. Li et al., Simulation and design of various configurations of silicon detectors for high irradiation 
tolerance up to $6 \times 10^{1} 4 \mathrm{n} / \mathrm{cm}^{2}$ in LHC application, Nucl. Instrum. Meth. A409 (1998) 180 .

[51] H.S. Cho et al., Characterization of silicon pixel detectors with the $n^{+} / n / p^{+}$and double-sided multiguard ring structure before and after neutron irradiation, IEEE Trans. Nucl. Sci. 47 (2000) 772.

[52] D. Bortoletto et al., Radiation damage studies of multi-guard ring p-type bulk diodes, Nucl. Instrum. Meth. A435 (1999) 178.

[53] S.I. Parker, Performance of 3D architecture silicon sensors after intenseproton irradiation, UH 511-959-00.

[54] Z. Li et al., Novel prototype Si detector development and processing at BNL, Nucl. Instrum. Meth. A478 (2002) 303.

[55] A. Roy, G. Bolla, D. Bortoletto and Z. Li, Semi-3D silicon detector and initial results of its performance, Nucl. Instrum. Meth. A552 (2005) 112.

[56] C. Da Via, 3D detectors 2 , talk at the $3^{\text {rd }}$ Workshop on Advanced Silicon Radiation Detectors (3D and P-type Technologies), April 14-16, Barcelona, Spain (2008).

[57] C. Piemonte, M. Boscardin, S. Ronchin, N. Zorzi and G.F. Dalla Betta, Development of 3D detectors featuring columnar electrodes of the same doping type, Nucl. Instrum. Meth. A541 (2005) 441.

[58] Z. Li et al., Development, simulation and processing of new 3D Si detectors, Nucl. Instrum. Meth. A 583 (2007) 139.

[59] G. Pellegrini et al., First double-sided 3D detectors fabricated at CNM-IMB, Nucl. Instrum. Meth. A592 (2008) 38.

[60] Z. Li et al., Cryogenic Si detectors for ultra radiation hardness in SLHC environment, Nucl. Instrum. Meth. A 579 (2007) 775.

[61] X. Rouby et al., RD39 status report 2008, CERN RD39 SR-2008.

[62] Z. Li et al., Detector recovery/improvement via elevated-temperature-annealing (DRIVE) - A new approach for Si detector applications in high radiation environment in SLHC, IEEE Trans. Nucl. Sci. 53 (2006) 1551. 